\title{
The Existence of Positive Solutions for Boundary Value Problem of the Fractional Sturm-Liouville Functional Differential Equation
}

\author{
Yanan Li, Shurong Sun, Zhenlai Han, and Hongling Lu \\ School of Mathematical Sciences, University of Jinan, Jinan, Shandong 250022, China \\ Correspondence should be addressed to Shurong Sun; sshrong@163.com
}

Received 25 April 2013; Accepted 20 June 2013

Academic Editor: Bashir Ahmad

Copyright @ 2013 Yanan Li et al. This is an open access article distributed under the Creative Commons Attribution License, which permits unrestricted use, distribution, and reproduction in any medium, provided the original work is properly cited.

We study boundary value problems for the following nonlinear fractional Sturm-Liouville functional differential equations involving the Caputo fractional derivative: ${ }^{C} D^{\beta}\left(p(t){ }^{C} D^{\alpha} u(t)\right)+f(t, u(t-\tau), u(t+\theta))=0, t \in(0,1),{ }^{C} D^{\alpha} u(0)={ }^{C} D^{\alpha} u(1)=$ $\left({ }^{C} D^{\alpha} u(0)\right)^{\prime \prime}=0, a u(t)-b u^{\prime}(t)=\eta(t), t \in[-\tau, 0], c u(t)+d u^{\prime}(t)=\xi(t), t \in[1,1+\theta]$, where ${ }^{C} D^{\alpha},{ }^{C} D^{\beta}$ denote the Caputo fractional derivatives, $f$ is a nonnegative continuous functional defined on $C([-\tau, 1+\theta], \mathbb{R}), 1<\alpha \leq 2,2<\beta \leq 3,0<\tau, \theta<1 / 4$ are suitably small, $a, b, c, d>0$, and $\eta \in C([-\tau, 0],[0, \infty)), \xi \in C([1,1+\theta],[0, \infty))$. By means of the Guo-Krasnoselskii fixed point theorem and the fixed point index theorem, some positive solutions are obtained, respectively. As an application, an example is presented to illustrate our main results.

\section{Introduction}

Fractional calculus is a branch of mathematics, it is an emerging field in the area of the applied mathematics that deals with derivatives and integrals of arbitrary orders as well as with their applications. The origins can be traced back to the end of the seventeenth century. During the history of fractional calculus, it was reported that the pure mathematical formulations of the investigated problems started to be addressed with more applications in various fields. With the help of fractional calculus, we can describe natural phenomena and mathematical models more accurately. Therefore, fractional differential equations have received much attention, and the theory and its applications have been greatly developed; see [1-6].

Recently, there have been many papers focused on boundary value problems of fractional ordinary differential equations [7-19] and initial value problems of fractional functional differential equations [10,20-27]. But the results dealing with the boundary value problems of fractional functional differential equations with delay are relatively scarce [28-32]. It is well known that in practical problems, the behavior of systems not only depends on the status just at the present but also on the status in the past [33]. Thus, in many cases, we must consider fractional functional differential equations with delay in order to solve practical problems. Consequently, our aim in this paper is to study the existence of solutions for boundary value problems of fractional functional differential equations.

In 2005, by means of the fixed point index theorem, Bai and $\mathrm{Ma}$ [34] established some criteria for the existence of solutions for the boundary value problem expressed by second order differential equations with delay:

$$
\begin{aligned}
& -\left(p(t) y^{\prime}(t)\right)^{\prime}+q(t) y(t) \\
& \quad=f(t, y(t-\tau), y(t+a)), \quad t \in(0,1), \\
& \alpha y(t)-\beta y^{\prime}(t)=\eta(t), \quad t \in[-\tau, 0], \\
& \gamma y(t)+\delta y^{\prime}(t)=\xi(t), \quad t \in[1,1+\varphi],
\end{aligned}
$$

where $0<\tau, \varphi<1 / 4$ are suitably small and $p \in C^{1}([0,1]$, $(0, \infty)), q \in C([0,1],[0, \infty)), q \leq 0$. 
In 2011, Li et al. [21] investigated the existence of positive solutions for the nonlinear Caputo fractional functional differential equation:

$$
D_{0+}^{\alpha} u(t)+a(t) f\left(u_{t}\right)=0, \quad 0<t<1,1<\alpha \leq 2,
$$

where $D_{0+}^{\alpha}$ is the Caputo fractional order derivative, subject to the following boundary conditions:

$$
\begin{gathered}
-a u(t)+b u^{\prime}(t)=\xi(t), \quad t \in[-\tau, 0], \\
c u(t)+d u^{\prime}(t)=\eta(t), \quad t \in[1,1+\beta] .
\end{gathered}
$$

They obtained the existence results of positive solutions by using some fixed point theorems.

In 2012, Zhao et al. [22] studied the existence of positive solutions for the nonlinear Caputo fractional functional differential equation:

$$
\begin{gathered}
D_{0+}^{q} y(t)+r(t) f\left(y_{t}\right)=0, \quad \forall t \in(0,1), q \in(n-1, n], \\
y^{(i)}(0)=0, \quad 0 \leq i \leq n-3, \\
\alpha y^{(n-2)}(t)-\beta y^{(n-1)}(t)=\eta(t), \quad t \in[-\tau, 0], \\
\gamma y^{(n-2)}(t)+\delta y^{(n-1)}(t)=\xi(t), \quad t \in[1,1+a] .
\end{gathered}
$$

By constructing a special cone and using the Guo-Krasnoselskii fixed point theorem, they obtained the existing results.

Motivated by the works above, in this paper, we study the existence of positive solutions of boundary value problems for nonlinear fractional functional differential equation:

$$
\begin{aligned}
& { }^{C} D^{\beta}\left(p(t){ }^{C} D^{\alpha} u(t)\right) \\
& +f(t, u(t-\tau), u(t+\theta))=0, \quad t \in(0,1), \\
& { }^{C} D^{\alpha} u(0)={ }^{C} D^{\alpha} u(1)=\left({ }^{C} D^{\alpha} u(0)\right)^{\prime \prime}=0, \\
& a u(t)-b u^{\prime}(t)=\eta(t), \quad t \in[-\tau, 0], \\
& c u(t)+d u^{\prime}(t)=\xi(t), \quad t \in[1,1+\theta],
\end{aligned}
$$

where ${ }^{C} D^{\alpha},{ }^{C} D^{\beta}$ denote the Caputo fractional derivatives, $f$ is a nonnegative continuous functional defined on $C([-\tau, 1+$ $\theta], \mathbb{R}), 1<\alpha \leq 2,2<\beta \leq 3,0<\tau, \theta<1 / 4$ are suitably small, $a, b, c, d>0$ with $\rho:=a c+a d+b c>0$ and $\eta \in$ $C([-\tau, 0],[0, \infty)), \xi \in C([1,1+\theta],[0, \infty))$ with $\eta(0)=\xi(1)=$ 0 , and $p$ is a positive measurable continuous function defined on $[0,1]$ and satisfies the following condition:

$$
0<\int_{E} \frac{G(s, s)}{p(s)} d s \leq \int_{0}^{1} \frac{G(s, s)}{p(s)} d s<+\infty,
$$

where $E=\{t \in[0,1] \mid 0 \leq t+a \leq 1,-\tau \leq a \leq \theta\}, p(0)=$ $p(1)=l$, and $l$ is a constant.

When $\alpha=\beta=1$, problem (5) is reduced to the problem of second order differential equations with delay and has been studied by Bai and $\mathrm{Ma}$ [34]. To the best of our knowledge, no one has studied the existence of positive solutions for boundary value problem (5). Key tools in finding our main results are fixed point index theorem and the GuoKrasnoselskii fixed point theorem, and our main results of this paper are to extend and supplement some results in $[21,22,34]$.

The paper is organized as follows. In Section 2, we will introduce some definitions and lemmas to prove our main results. In Section 3, we investigate the existence of positive solution for boundary value problems (5) by the Guo-Krasnoselskii fixed point theorem and the fixed point index theorem. As an application, an example is presented to illustrate our main results.

\section{Preliminaries}

In the following section, we introduce the definitions and lemmas which are used throughout the paper.

Definition 1 (see [4]). The fractional integral of order $\alpha(\alpha>$ $0)$ of a function $f:\left(t_{0},+\infty\right) \rightarrow \mathbb{R}$ is given by

$$
I^{\alpha} f(t)=\frac{1}{\Gamma(\alpha)} \int_{t_{0}}^{t} \frac{f(s)}{(t-s)^{1-\alpha}} d s, \quad t>t_{0},
$$

where $\Gamma(\cdot)$ is the gamma function, provided that the right side is pointwise defined on $\left(t_{0},+\infty\right)$.

Definition 2 (see [4]). The Caputo fractional derivative of order $\alpha(n-1<\alpha<n)$ of a function $f:\left(t_{0},+\infty\right) \rightarrow \mathbb{R}$ is given by

$$
{ }^{C} D^{\alpha} f(t)=\frac{1}{\Gamma(n-\alpha)} \int_{t_{0}}^{t} \frac{f^{(n)}(s)}{(t-s)^{\alpha+1-n}} d s, \quad t>t_{0},
$$

where $\Gamma(\cdot)$ is the gamma function, provided that the right side is pointwise defined on $\left(t_{0},+\infty\right)$.

Obviously, the Caputo derivative for every constant function is equal to zero.

From the definition of the Caputo derivative, we can acquire the following statements.

Lemma 3 (see [5]). Let $f(t) \in L^{1}\left[t_{0}, \infty\right)$. Then,

$$
{ }^{C} D^{\alpha}\left(I^{\alpha} f(t)\right)=f(t), \quad t>t_{0}, 0<\alpha<1 .
$$

Lemma 4 (see [5]). Let $\alpha>0$. Then,

$$
I^{\alpha C} D^{\alpha} f(t)=f(t)-c_{1}-c_{2} t-\cdots-c_{n} t^{n-1},
$$

for some $c_{i} \in \mathbb{R}, i=1,2, \ldots, n$, where $n$ is the smallest integer greater than or equal to $\alpha$.

Assume that $x_{0}$ is the solution of (5) with $f \equiv 0$; then it can be expressed as

$$
x_{0}(t)= \begin{cases}x_{0}(-\tau ; t) & t \in[-\tau, 0] \\ 0 & t \in[0,1] \\ x_{0}(\theta ; t) & t \in[1,1+\theta],\end{cases}
$$


where

$$
\begin{gathered}
x_{0}(-\tau ; t)=\frac{1}{b} e^{a t / b} \int_{t}^{0} e^{-a s / b} \eta(s) d s \\
x_{0}(\theta ; t)=\frac{1}{d} e^{-c t / d} \int_{1}^{t} e^{c s / d} \xi(s) d s .
\end{gathered}
$$

Next, we introduce the Green function of boundary value problems for fractional functional differential equations.

Lemma 5. Let $1<\alpha \leq 2$ and $f:[0,1] \times(0, \infty) \times(0, \infty) \rightarrow \mathbb{R}$ be continuous. Then, the boundary value problem for fractional functional differential equation

$$
\begin{gathered}
{ }^{C} D^{\alpha} u(t)+f(t, u(t-\tau), u(t+\theta))=0, \quad t \in(0,1), \\
a u(t)-b u^{\prime}(t)=\eta(t), \quad t \in[-\tau, 0], \\
c u(t)+d u^{\prime}(t)=\xi(t), \quad t \in[1,1+\theta]
\end{gathered}
$$

has a unique solution:

$$
u(t)= \begin{cases}u(-\tau ; t) & t \in[-\tau, 0] \\ \int_{0}^{1} G(t, s) f(s, u(s-\tau), u(s+\theta)) d s & t \in[0,1] \\ u(\theta ; t) & t \in[1,1+\theta]\end{cases}
$$

where

$$
\begin{gathered}
u(-\tau ; t)=e^{(a / b) t}\left(\frac{1}{b} \int_{t}^{0} e^{-(a / b) s} \eta(s) d s+u(0)\right) \\
u(\theta ; t)=e^{-(c / d) t}\left(\frac{1}{d} \int_{1}^{t} e^{(c / d) s} \xi(s) d s+u(1)\right) \\
G(t, s)=\frac{1}{\Gamma(\alpha)} \begin{cases}g_{1}(t, s) & 0 \leq s \leq t \leq 1 \\
g_{2}(t, s) & 0 \leq t \leq s \leq 1,\end{cases} \\
g_{1}(t, s)=-(t-s)^{\alpha-1}+\frac{b+a t}{b c+a c+a d} \\
\times\left(c(1-s)^{\alpha-1}+d(\alpha-1)(1-s)^{\alpha-2}\right), \\
g_{2}(t, s)=\frac{b+a t}{b c+a c+a d}\left(c(1-s)^{\alpha-1}+d(\alpha-1)(1-s)^{\alpha-2}\right) .
\end{gathered}
$$

Proof. It is easy to know that $u(t)=u(-\tau ; t)$ for $t \in[-\tau, 0]$ and $u(t)=u(\theta ; t)$ for $t \in[1,1+\theta]$. From (13), we know that

$$
I^{\alpha C} D^{\alpha} u(t)=-I^{\alpha} f(t, u(t-\tau), u(t+\theta)), \quad 1<\alpha \leq 2 .
$$

From Lemma 4, we have

$$
\begin{aligned}
u(t)= & -\frac{1}{\Gamma(\alpha)} \int_{0}^{t}(t-s)^{\alpha-1} f(s, u(s-\tau), u(s+\theta)) d s \\
& +c_{1}+c_{2} t .
\end{aligned}
$$

Then, we get that

$$
\begin{aligned}
u^{\prime}(t)= & -\frac{1}{\Gamma(\alpha-1)} \int_{0}^{t}(t-s)^{\alpha-2} f(s, u(s-\tau), u(s+\theta)) d s \\
& +c_{2} .
\end{aligned}
$$

According to (14) and conditions $\eta(0)=\xi(1)=0$, we imply that

$$
a u(0)-b u^{\prime}(0)=0, \quad c u(1)+d u^{\prime}(1)=0 .
$$

Then, we obtain

$$
\begin{aligned}
& c_{2}= \frac{a}{a c+b c+a d} \\
& \times\left(\frac{c}{\Gamma(\alpha)} \int_{0}^{1}(1-s)^{\alpha-1} f(s, u(s-\tau), u(s+\theta)) d s\right. \\
&+\frac{d}{\Gamma(\alpha-1)} \\
&\left.\times \int_{0}^{1}(1-s)^{\alpha-2} f(s, u(s-\tau), u(s+\theta)) d s\right), \\
& \frac{b}{a c+}+b c+a d \\
& \times\left(\frac{c}{\Gamma(\alpha)} \int_{0}^{1}(1-s)^{\alpha-1} f(s, u(s-\tau), u(s+\theta)) d s\right. \\
& \quad+\frac{d}{\Gamma(\alpha-1)} \\
&\left.\quad \times \int_{0}^{1}(1-s)^{\alpha-2} f(s, u(s-\tau), u(s+\theta)) d s\right) .
\end{aligned}
$$

Therefore,

$$
\begin{aligned}
u(t)= & -\frac{1}{\Gamma(\alpha)} \int_{0}^{t}(t-s)^{\alpha-1} f(s, u(s-\tau), u(s+\theta)) d s \\
& +\frac{a t+b}{a c+b c+a d} \\
& \times\left(\frac{c}{\Gamma(\alpha)} \int_{0}^{1}(1-s)^{\alpha-1} f(s, u(s-\tau), u(s+\theta)) d s\right. \\
& +\frac{d}{\Gamma(\alpha-1)} \\
& \left.\times \int_{0}^{1}(1-s)^{\alpha-2} f(s, u(s-\tau), u(s+\theta)) d s\right) \\
= & \int_{0}^{1} G(t, s) f(s, u(s-\tau), u(s+\theta)) d s
\end{aligned}
$$

where $G(t, s)$ is defined by (17). The proof is completed. 
Lemma 6. Let $1<\alpha \leq 2,2<\beta \leq 3$ and $f:[0,1] \times$ $(0, \infty) \times(0, \infty) \rightarrow \mathbb{R}$ be continuous. Then, the boundary value problem for fractional functional differential equation

$$
\begin{aligned}
& { }^{C} D^{\beta}\left(p(t){ }^{C} D^{\alpha} u(t)\right) \\
& =f(t, u(t-\tau), u(t+\theta)), \quad t \in(0,1), \\
& { }^{C} D^{\alpha} u(0)={ }^{C} D^{\alpha} u(1)=\left({ }^{C} D^{\alpha} u(0)\right)=0, \\
& \alpha u(t)-b u^{\prime}(t)=\eta(t), \quad t \in[-\tau, 0], \\
& c u(t)+d u^{\prime}(t)=\xi(t), \quad t \in[1,1+\theta]
\end{aligned}
$$

has a unique solution:

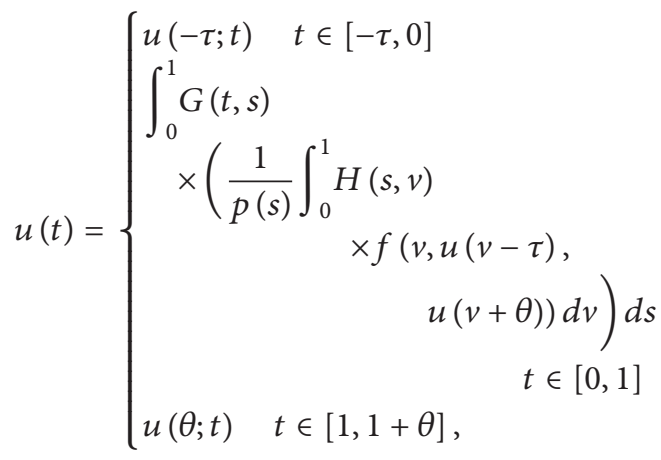

where $G(t, s)$ is defined by (17), and

$$
H(s, v)=\frac{1}{\Gamma(\beta)} \begin{cases}s(1-v)^{\beta-1}-(s-v)^{\beta-1} & 0 \leq v \leq s \leq 1 \\ s(1-v)^{\beta-1} & 0 \leq s \leq v \leq 1 .\end{cases}
$$

Proof. From (25), we know that

$$
I^{\beta C} D^{\beta}\left(p(t){ }^{C} D^{\alpha} u(t)\right)=I^{\beta} f(t, u(t-\tau), u(t+\theta)) .
$$

From Lemma 4, we have

$$
\begin{aligned}
{ }^{C} D^{\alpha} u(t)= & \frac{1}{p(t) \Gamma(\beta)} \\
& \times \int_{0}^{t}(t-v)^{\beta-1} f(v, u(v-\tau), u(v+\theta)) d v \\
& +\frac{c_{1}+c_{2} t+c_{3} t^{2}}{p(t)} .
\end{aligned}
$$

According to the conditions ${ }^{C} D^{\alpha} u(0)={ }^{C} D^{\alpha} u(1)=$ $\left({ }^{C} D^{\alpha} u(0)\right)^{\prime \prime}=0$ and $p(0)=p(1)=l$, we derive that

$$
\begin{gathered}
c_{1}=0, \quad c_{3}=0, \\
c_{2}=-\frac{1}{\Gamma(\beta)} \int_{0}^{1}(1-v)^{\beta-1} f(v, u(v-\tau), u(v+\theta)) d v .
\end{gathered}
$$

Therefore,

$$
\begin{aligned}
p(t) & { }^{C} D^{\alpha} u(t) \\
= & \frac{1}{\Gamma(\beta)} \int_{0}^{t}(t-v)^{\beta-1} f(v, u(v-\tau), u(v+\theta)) d v \\
& -\frac{t}{\Gamma(\beta)} \int_{0}^{1}(1-v)^{\beta-1} f(v, u(v-\tau), u(v+\theta)) d v \\
= & \frac{1}{\Gamma(\beta)} \int_{0}^{t}(t-v)^{\beta-1} f(v, u(v-\tau), u(v+\theta)) d v \\
& -\frac{t}{\Gamma(\beta)} \int_{0}^{t}(1-v)^{\beta-1} f(v, u(v-\tau), u(v+\theta)) d v \\
& -\frac{t}{\Gamma(\beta)} \int_{t}^{1}(1-v)^{\beta-1} f(v, u(v-\tau), u(v+\theta)) d v \\
= & -\int_{0}^{1} H(t, v) f(v, u(v-\tau), u(v+\theta)) d v .
\end{aligned}
$$

Thus,

$$
p(t)^{C} D^{\alpha} u(t)+\int_{0}^{1} H(t, v) f(v, u(v-\tau), u(v+\theta)) d v=0 .
$$

Then boundary value problem (25) is equivalent to the following problem:

$$
\begin{aligned}
&{ }^{C} D^{\alpha} u(t)+\frac{1}{p(t)} \\
& \times \int_{0}^{1} H(t, v) f(v, u(v-\tau), u(v+\theta)) d v=0, \quad t \in[0,1], \\
& \quad a u(t)-b u^{\prime}(t)=\eta(t), \quad t \in[-\tau, 0], \\
& \quad c u(t)+d u^{\prime}(t)=\xi(t), \quad t \in[1,1+\theta] .
\end{aligned}
$$

Lemma 5 implies that boundary value problem (33) has a unique solution:

$$
u(t)=\left\{\begin{array}{c}
u(-\tau ; t) \quad t \in[-\tau, 0] \\
\int_{0}^{1} G(t, s) \\
\quad \times\left(\frac{1}{p(s)} \int_{0}^{1} H(s, v)\right. \\
\quad \times f(v, u(v-\tau), u(v+\theta)) d v) d s \\
u(\theta ; t) \quad t \in[1,1+\theta],
\end{array}\right.
$$

where $G(t, s)$ and $H(s, v)$ are defined as (17) and (27), respectively. The proof is completed.

The following properties of the Green function play important roles in this paper. 
Lemma 7 (see [21]). The function $G(t, s)$ defined by (17) satisfies the following conditions:

(1) $G(t, s)$ is continuous on $[0,1] \times[0,1]$;

(2) for $b>((2-\alpha) /(\alpha-1)) a$, we have $G(t, s)>0$ for $t, s \in(0,1)$

(3) $G(t, s) \leq G(s, s)$ for $t, s \in(0,1)$;

(4) there exist positive numbers $z, z^{*}$ such that

$$
z Y(s) \leq G(t, s) \leq z^{*} Y(s),
$$

where $Y(s)=(b+a s)\left((c / \Gamma(\alpha))(1-s)^{\alpha-1}+(d / \Gamma(\alpha-\right.$ 1)) $\left.(1-s)^{\alpha-2}\right)$.

Remark 8. From the definition of $G(t, s)$ and $Y(s)$, we know that $G(s, s)=Y(s) /(a c+a d+b c)$. Furthermore, its easy to get from the proof of Lemma 3.3 in [21] that there exists a positive number $\gamma_{1}<1$ such that

$$
G(t, s) \geq \gamma_{1} G(s, s) \quad \forall t, s \in(0,1),
$$

where $\gamma_{1}=z(a c+a d+b c)$.

Lemma 9 (see [18]). The function $H(s, v)$ defined by (27) satisfies the following conditions:

(1) $H(s, v)>0$ for $s, v \in(0,1)$;

(2) there exists a positive function $\gamma_{2}(v) \in C(0,1)$ for $v \in$ $(0,1)$ such that

$$
\begin{gathered}
\min _{1 / 4 \leq s \leq 3 / 4} H(s, v) \geq \gamma_{2}(v) M(v), \\
\max _{0 \leq s \leq 1} H(s, v) \leq M(v),
\end{gathered}
$$

where

$$
\begin{aligned}
& M(v) \\
& =H\left(s_{0}, v\right) \\
& =\frac{(\beta-2)(\beta-1)^{2 /(2-\beta)}(1-v)^{(\beta-1)^{2} /(\beta-2)}+v(1-v)^{\beta-1}}{\Gamma(\beta)}, \\
& s_{0}=(\beta-1)^{-1 /(\beta-2)}(1-v)^{(\beta-1) /(\beta-2)}+v, \quad s_{0} \in[v, 1] .
\end{aligned}
$$

The following lemmas are fundamental in proof of our main results.

Lemma 10 (see [35]). Let $E$ be a Banach space, and let $K \subset E$ be a cone. Assume that $\Omega_{1}, \Omega_{2}$ are open and bounded subsets of $E$ with $0 \in \Omega_{1}, \bar{\Omega}_{1} \subset \Omega_{2}$, and let $T: K \cap\left(\bar{\Omega}_{2} \backslash \Omega_{1}\right) \rightarrow K$ be a completely continuous operator such that

(i) $\|T u\| \leq\|u\|, u \in K \cap \partial \Omega_{1}$, and $\|T u\| \geq\|u\|, u \in$ $K \cap \partial \Omega_{2}$; (ii) $\|T u\| \geq\|u\|, u \in K \cap \partial \Omega_{1}$, and $\|T u\| \leq\|u\|, u \in$ $K \cap \partial \Omega_{2}$.

Then, $T$ has a fixed point in $K \cap\left(\bar{\Omega}_{2} \backslash \Omega_{1}\right)$.

Lemma 11 (see [36]). Assume that $X$ is a Banach space and $K \subset X$ is a cone. Let $K_{r}=\{u \in K ;\|u\|<r\}$. Furthermore, assume that $T: K \rightarrow K$ is compact and $T u \neq u$ for $u \in \partial K_{r}=$ $\{u \in K ;\|u\|=r\}$. Thus, one has the following conclusions:

(i) if $\|u\| \leq\|T u\|$ for $u \in \partial K_{r}$, then $i\left(T, K_{r}, K\right)=0$;

(ii) if $\|u\| \geq\|T u\|$ for $u \in \partial K_{r}$, then $i\left(T, K_{r}, K\right)=1$.

\section{Main Results}

In this section, we discuss the existence of positive solutions for boundary value problem (5).

Let $X=C[-\tau, 1+\theta]$ be a Banach space with the maximum norm $\|u\|_{[-\tau, 1+\theta]}=\max _{-\tau \leq t \leq 1+\theta}|u(t)|$ for $u \in X$. Let $K$ be a cone in $X$ defined by

$$
\begin{array}{r}
K=\{u \in X ; u(t) \geq 0 \text { for } t \in[-\tau, 1+\theta] ; \\
\left.u(t) \geq \gamma_{1}\|u\| \text { for each } t \in[0,1]\right\} .
\end{array}
$$

Let $x(t)=u(t)-x_{0}(t)$. Noting that $x(t) \equiv u(t)$ for $t \epsilon$ $[0,1]$, we have

$$
x(t)=\left\{\begin{array}{c}
e_{0}^{a t / b} x(0) \quad t \in[-\tau, 0] \\
\times\left(\frac{1}{p(s)} \int_{0}^{1} H(s, v)\right. \\
\quad \times f\left(v,\left(x+x_{0}\right)(v-\tau),\right. \\
\left.\left.\left(x+x_{0}\right)(v+\theta)\right) d v\right) d s \\
\quad t \in[0,1] \\
\quad t \in[1,1+\theta],
\end{array}\right.
$$

where

$$
\begin{aligned}
& \left(x+x_{0}\right)(v-\tau):=x(v-\tau)+x_{0}(v-\tau), \\
& \left(x+x_{0}\right)(v+\theta):=x(v+\theta)+x_{0}(v+\theta) .
\end{aligned}
$$


Define an operator $T$ on $K$ as follows:

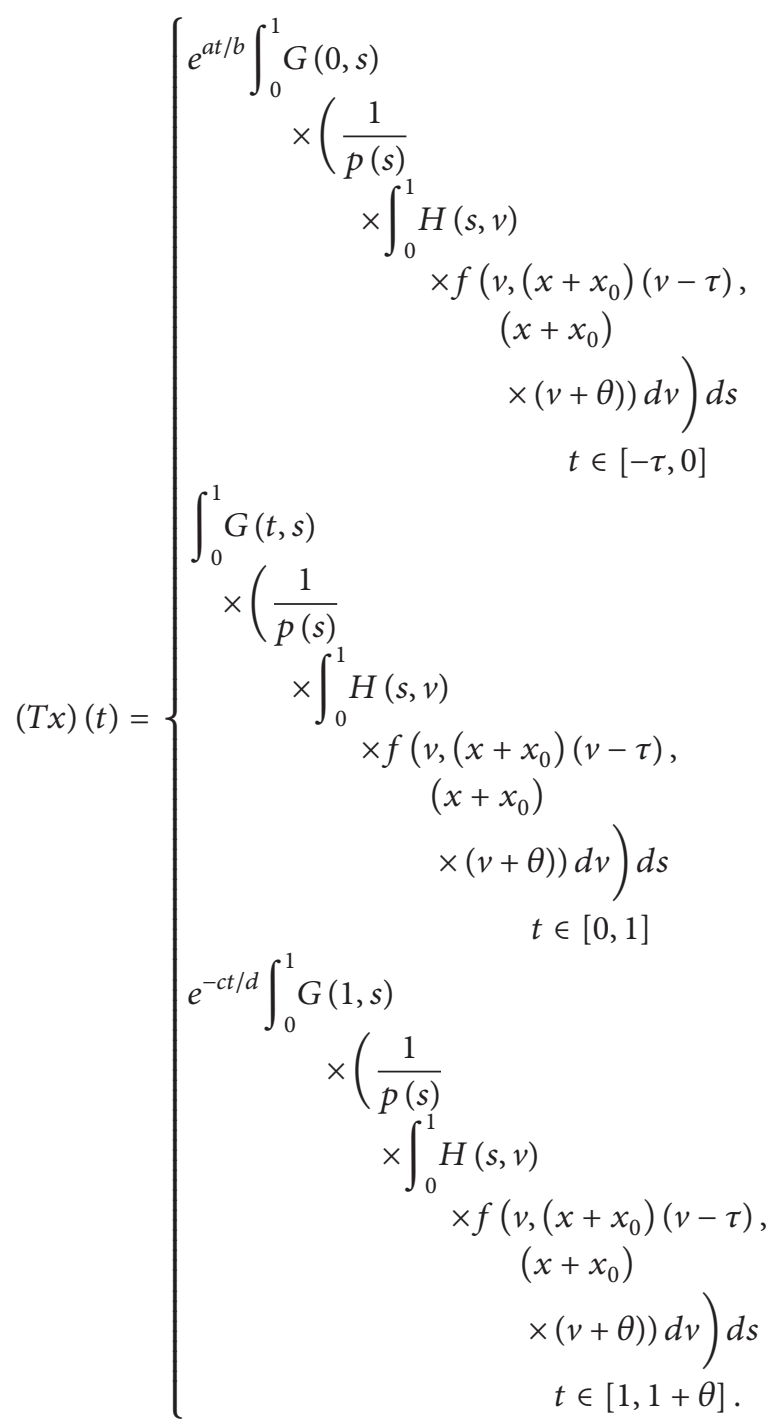

Lemma 12. The operator $T: K \rightarrow K$ is completely continuous.

Proof. For $x \in K$, we have $(T x)(t) \geq 0, t \in[-\tau, 1+\theta]$ from properties of $G(t, s)$ and $H(s, v)$. It follows from (55) that we have for $t \in[-\tau, 0]$,

$$
(T x)(t) \leq(T x)(0)
$$

and for $t \in[1,1+\theta]$,

$$
(T x)(t) \leq(T x)(1) .
$$

Hence, we get that

$$
\|T x\|_{[-\tau, 1+\theta]}=\|T x\|_{[0,1]} .
$$

By Lemma 7 and Remark 8, we have

$$
\begin{aligned}
& \|(T x)(t)\|_{[0,1]} \\
& \leq \int_{0}^{1} G(s, s) \\
& \quad \times\left(\frac{1}{p(s)} \int_{0}^{1} H(s, v)\right. \\
& \times f\left(v,\left(x+x_{0}\right)(v-\tau),\right. \\
& \left.\left.\quad\left(x+x_{0}\right)(v+\theta)\right) d v\right) d s,
\end{aligned}
$$

and

$$
\begin{aligned}
& (T x)(t) \geq \int_{0}^{1} \gamma_{1} G(s, s) \\
& \quad \times\left(\frac{1}{p(s)} \int_{0}^{1} H(s, v)\right. \\
& \quad \times f\left(v,\left(x+x_{0}\right)(v-\tau),\right. \\
& \left.\left.\quad\left(x+x_{0}\right)(v+\theta)\right) d v\right) d s \\
& \geq \gamma_{1}\|(T x)(t)\|_{[0,1]} \\
& =\gamma_{1}\|(T x)(t)\| .
\end{aligned}
$$

Thus, $T(K) \subseteq K$.

Let $\Omega$ be a bounded subset in $K$, that is, there exists a positive constant $\zeta>0$ such that $\|x\| \leq \zeta$, for all $x \in \Omega$. Let $Q:=\max _{0 \leq v \leq 1,0 \leq x \leq \zeta}\left|f\left(v,\left(x+x_{0}\right)(v-\tau),\left(x+x_{0}\right)(v+\theta)\right)\right|+1$. Then, for $x \in \Omega$, in view of Lemma 9 , we have

$$
\begin{aligned}
(T x)(t)=\int_{0}^{1} G(t, s) & \\
& \times\left(\frac{1}{p(s)} \int_{0}^{1} H(s, v)\right. \\
& \times f(v, u(v-\tau), u(v+\theta)) d v) d s \\
\leq & Q \int_{0}^{1} G(s, s)\left(\frac{1}{p(s)} \int_{0}^{1} M(v) d v\right) d s .
\end{aligned}
$$

Hence, $T(\Omega)$ is bounded in $K$.

Now, we divide three cases to prove that $T(\Omega)$ is equicontinuous. 
Case 1. If $u \in \Omega, 0<t<1$.

Then,

$$
\begin{aligned}
\left|(T x)^{\prime}(t)\right| \leq \mid & -\frac{Q}{\Gamma(\alpha-1)} \\
& \times \int_{0}^{1}(t-s)^{\alpha-2}\left(\frac{1}{p(s)} \int_{0}^{1} M(v) d v\right) d s \\
& +\frac{a Q}{\Gamma(\alpha)(b c+a c+a d)} \\
& \times \int_{0}^{1}\left(c(1-s)^{\alpha-1}+d(\alpha-1)(1-s)^{\alpha-2}\right) \\
:=Q_{1} . & \times\left(\frac{1}{p(s)} \int_{0}^{1} M(v) d v\right) d s \mid
\end{aligned}
$$

Case 2. If $u \in \Omega,-\tau \leq t \leq 0$.

Then,

$$
\begin{aligned}
& \left|(T x)^{\prime}(t)\right| \\
& =\mid \frac{a}{b} e^{a t / b} \int_{0}^{1} G(0, s) \\
& \quad \times\left(\frac{1}{p(s)} \int_{0}^{1} H(s, v)\right. \\
& \qquad \times f(v, u(v-\tau),(v+\theta)) d v) d s \mid \\
& \leq \frac{a Q}{b} \int_{0}^{1} G(s, s)\left(\frac{1}{p(s)} \int_{0}^{1} M(v) d v\right) d s \\
& :=Q_{2} .
\end{aligned}
$$

Case 3. If $u \in \Omega, 1 \leq t \leq 1+\theta$.

Then,

$$
\begin{aligned}
& \left|(T x)^{\prime}(t)\right| \\
& =\mid-\frac{c}{d} e^{-c t / d} \\
& \quad \times \int_{0}^{1} G(1, s) \\
& \quad \times\left(\frac{1}{p(s)} \int_{0}^{1} H(s, v)\right. \\
& \quad \times f(v, u,(v-\tau), u(v+\theta)) d v) d s \mid \\
& \leq \frac{c Q}{d} \int_{0}^{1} G(s, s)\left(\frac{1}{p(s)} \int_{0}^{1} M(v) d v\right) d s:=Q_{3} .
\end{aligned}
$$

Let $\delta_{1}=\epsilon_{1} / \max \left\{Q_{1}, Q_{2}, Q_{3}\right\}$. Then, for $t_{1}, t_{2} \in[-\tau, 1+\theta]$, $\left|t_{2}-t_{1}\right|<\delta_{1}$, we obtain

$$
\begin{aligned}
\left|(T x)\left(t_{1}\right)-(T x)\left(t_{2}\right)\right| & \leq \int_{t_{1}}^{t_{2}}\left|(T x)^{\prime}(t)\right| d t \\
& \leq \max \left\{Q_{1}, Q_{2}, Q_{3}\right\}\left|t_{2}-t_{1}\right|<\epsilon_{1} .
\end{aligned}
$$

Thus, $T(\Omega)$ is equicontinuous.

Next, we show that $T: K \rightarrow K$ is continuous. For any $x_{n}, x \in K, n=1,2 \ldots$ with $\left\|x_{n}-x\right\|_{[-\tau, 1+\theta]} \rightarrow 0$ as $n \rightarrow \infty$. Then, for $t \in[0,1]$, we have

$$
\begin{aligned}
& \left|\left(T x_{n}\right)(t)-(T x)(t)\right| \\
& \leqslant \sup _{v \in[0,1]} \mid f\left(v,\left(x_{n}+x_{0}\right)(v-\tau),\left(x_{n}+x_{0}\right)(v+\theta)\right) \\
& -f\left(v,\left(x+x_{0}\right)(v-\tau),\left(x+x_{0}\right)(v+\theta)\right) \mid \\
& \times \int_{0}^{1} G(s, s)\left(\frac{1}{p(s)} \int_{0}^{1} M(v) d v\right) d s .
\end{aligned}
$$

This implies that $\left\|T x_{n}-T x\right\|_{[-\tau, 1+\theta]} \rightarrow 0$ as $n \rightarrow \infty$. Hence, $T$ is continuous. According to the Ascoli-Arzelà Theorem, $T$ is completely continuous. The proof is completed.

For convenience, we give some conditions, which will play roles in this paper for $f(t, \mu, \nu)$ as follows

$$
\begin{aligned}
& \left(H_{1}\right) \liminf _{\mu+\nu \rightarrow 0^{+}} \min _{t \in[0,1]} f(t, \mu, \nu) /(\mu+\nu)=\infty . \\
& \left(H_{2}\right) \lim \inf _{\mu+\nu \rightarrow+\infty} \min _{t \in[0,1]}(f(t, \mu, \nu)) /(\mu+\nu)=\infty . \\
& \left(H_{3}\right) \lim \sup _{\mu+\nu \rightarrow+\infty} \min _{t \in[0,1]}(f(t, \mu, \nu)) /(\mu+\nu)=0 .
\end{aligned}
$$

Lemma 13. Let $\left(H_{1}\right)$ and $\left(H_{2}\right)$ hold. Then, there exist positive numbers $0<r_{0}<R_{0}<\infty$ such that

$$
\begin{gathered}
i\left(T, K_{r}, K\right)=0 \quad \text { for } 0<r \leq r_{0}, \\
i\left(T, K_{R}, K\right)=0 \quad \text { for } R_{0} \leq R .
\end{gathered}
$$

Proof. Choose $L>0$ such that

$$
\gamma_{1} L \int_{1 / 4}^{3 / 4} G\left(\frac{1}{2}, s\right)\left(\frac{1}{p(s)} \int_{1 / 4}^{3 / 4} \gamma_{2}(v) M(v) d v\right) d s>\frac{1}{2} .
$$

By $\left(H_{1}\right)$, there is an $r_{0}>0$ such that $0<r \leq r_{0}$ implies that

$$
\begin{gathered}
f(\nu, \mu, v) \geq L(\mu+\nu), \\
\mu, v \geq 0, \quad \mu+v \leq 2 r, \quad v \in[0,1] .
\end{gathered}
$$

Thus, for $x \in \partial K_{r}$, we have

$$
\begin{aligned}
& r=\|x\| \geq x(v-\tau) \geq \gamma_{1}\|x\| \geq \gamma_{1} r, \quad v \in\left[\frac{1}{4}, \frac{3}{4}\right], \\
& r=\|x\| \geq x(v+\varphi) \geq \gamma_{1}\|x\| \geq \gamma_{1} r, \quad v \in\left[\frac{1}{4}, \frac{3}{4}\right] .
\end{aligned}
$$


In view of (55) and (56), we get that

$$
\begin{aligned}
& (T x)\left(\frac{1}{2}\right) \\
& \geq \int_{1 / 4}^{3 / 4} G\left(\frac{1}{2}, s\right) \\
& \times\left(\frac{1}{p(s)} \int_{1 / 4}^{3 / 4} H(s, v)\right. \\
& \times f\left(v,\left(x+x_{0}\right)(v-\tau),\right. \\
& \left.\left.\left(x+x_{0}\right)(v+\theta)\right) d v\right) d s \\
& \geq \int_{1 / 4}^{3 / 4} G\left(\frac{1}{2}, s\right) \\
& \times\left(\frac{1}{p(s)} \int_{1 / 4}^{3 / 4} \gamma_{2}(v) M(v)\right. \\
& \times f(v, x(v-\tau), x(v+\theta)) d v) d s \\
& \geq L \int_{1 / 4}^{3 / 4} G\left(\frac{1}{2}, s\right) \\
& \times\left(\frac{1}{p(s)} \int_{1 / 4}^{3 / 4} \gamma_{2}(v) M(v)\right. \\
& \times(x(v-\tau)+x(v+\theta)) d v) d s \\
& \geq 2 \gamma_{1} r L \int_{1 / 4}^{3 / 4} G\left(\frac{1}{2}, s\right)\left(\frac{1}{p(s)} \int_{1 / 4}^{3 / 4} \gamma_{2}(v) M(v) d v\right) d s \\
& >r=\|x\| .
\end{aligned}
$$

Hence, $\|T x\|>\|x\|$. It is obvious that $T x \neq x$ for $x \in \partial K_{r}$. Therefore, by Lemma 11 , we conclude that $i\left(T, K_{r}, K\right)=0$.

In the same way, for the same $L>0$ satisfying $(55),\left(H_{2}\right)$ implies that there is $R_{1}>0$ such that

$$
\begin{gathered}
f(\nu, \mu, \nu) \geq L(\mu+\nu), \\
\mu, v \geq 0, \quad \mu+\nu \geq R_{1}, \quad v \in[0,1] .
\end{gathered}
$$

Choose

$$
R_{0}>\max \left\{r, \frac{R_{1}}{2 \gamma_{1}}\right\} .
$$

Thus, for $x \in \partial K_{R}, R \geq R_{0}$, we have

$$
\begin{aligned}
R & =\|x\| \geq x(v-\tau) \geq \gamma_{1}\|x\| \geq \gamma_{1} R \\
& \geq \gamma_{1} R_{0}>\frac{R_{1}}{2}, \quad v \in\left[\frac{1}{4}, \frac{3}{4}\right], \\
R & =\|x\| \geq x(v+\theta) \geq \gamma_{1}\|x\| \geq \gamma_{1} R \\
& \geq \gamma_{1} R_{0}>\frac{R_{1}}{2}, \quad v \in\left[\frac{1}{4}, \frac{3}{4}\right] .
\end{aligned}
$$

In view of (55) and (59), we get that

$$
\begin{aligned}
& (T x)\left(\frac{1}{2}\right) \\
& \geq \int_{1 / 4}^{3 / 4} G\left(\frac{1}{2}, s\right) \\
& \times\left(\frac{1}{p(s)} \int_{1 / 4}^{3 / 4} H(s, v)\right. \\
& \times f\left(v,\left(x+x_{0}\right)(v-\tau),\right. \\
& \left.\left.\left(x+x_{0}\right)(v+\theta)\right) d v\right) d s \\
& \geq \int_{1 / 4}^{3 / 4} G\left(\frac{1}{2}, s\right) \\
& \times\left(\frac{1}{p(s)} \int_{1 / 4}^{3 / 4} \gamma_{2}(v) M(v)\right. \\
& \times f(v, x(v-\tau), x(v+\theta)) d v) d s \\
& \geq L \int_{1 / 4}^{3 / 4} G\left(\frac{1}{2}, s\right) \\
& \times\left(\frac{1}{p(s)} \int_{1 / 4}^{3 / 4} \gamma_{2}(v) M(v)\right. \\
& \times(x(v-\tau)+x(v+\theta)) d v) d s \\
& \geq 2 \gamma_{1} L R \int_{1 / 4}^{3 / 4} G\left(\frac{1}{2}, s\right) \\
& \times\left(\frac{1}{p(s)} \int_{1 / 4}^{3 / 4} \gamma_{2}(v) M(v) d v\right) d s \\
& >R=\|x\| .
\end{aligned}
$$

Therefore, $\|T x\|>\|x\|$. By Lemma 11, we conclude that $i(T$, $\left.K_{R}, K\right)=0$. The proof is completed.

Lemma 14. Let $\left(H_{3}\right)$ hold. Then, there exists a $0<R_{0}<\infty$ such that

$$
i\left(T, K_{R}, K\right)=1 \quad \text { for } R_{0} \leq R \text {. }
$$


Abstract and Applied Analysis

9

Proof. By $\left(H_{3}\right)$, for any

$$
0<\epsilon_{2}<\frac{1}{2}\left(\int_{0}^{1} G(s, s)\left(\frac{1}{p(s)} \int_{0}^{1} M(v) d v\right) d s\right)^{-1},
$$

there exists $R^{\prime}>0$ such that

$$
f(\nu, \mu, \nu) \leq \epsilon_{2}(\mu+\nu),
$$

$$
\mu, v \geq 0, \quad \mu+v \geq R^{\prime}, \quad v \in[0,1] .
$$

Setting

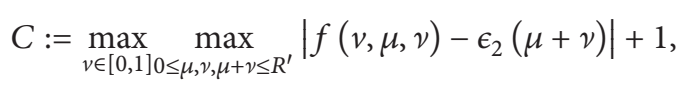

we get that

$$
f(v, \mu, \nu) \leq \epsilon_{2}(\mu+\nu)+C, \quad \mu, v \geq 0, v \in[0,1] .
$$

Choose

$$
R_{0} \geq \frac{\left(C+2 \epsilon_{2}\left\|x_{0}\right\|\right) \int_{0}^{1} G(s, s)\left((1 / p(s)) \int_{0}^{1} M(v) d v\right) d s}{\left(1-2 \epsilon_{2} \int_{0}^{1} G(s, s)\left((1 / p(s)) \int_{0}^{1} M(v) d v\right) d s\right)} .
$$

By (67), for $x \in \partial K_{R}, R \geq R_{0}$, we have

$$
(T x)(t)
$$

$$
\begin{aligned}
& =\int_{0}^{1} G(t, s) \\
& \times\left(\frac{1}{p(s)} \int_{0}^{1} H(s, v)\right. \\
& \times f\left(v,\left(x+x_{0}\right)(v-\tau),\right. \\
& \left.\left.\left(x+x_{0}\right)(v+\theta)\right) d v\right) d s
\end{aligned}
$$

$$
\begin{aligned}
\leq \int_{0}^{1} G(s, s) \\
\quad \times\left(\frac{1}{p(s)} \int_{0}^{1} M(v)\right.
\end{aligned}
$$

$$
\begin{gathered}
\times f\left(v,\left(x+x_{0}\right)(v-\tau)\right. \\
\left.\left.\left(x+x_{0}\right)(v+\theta)\right) d v\right) d s \\
\leq \int_{0}^{1} G(s, s) \quad \times\left(\epsilon _ { 2 } \left(\left(x+x_{0}\right)(v-\tau)\right.\right. \\
\times\left(\frac{1}{p(s)} \int_{0}^{1} M(v)\right. \\
\left.\left.\left.\leq \int_{0}^{1} G(s, s) \quad+\left(x+x_{0}\right)(v+\theta)\right)+C\right) d v\right) d s \\
\quad \times\left(\frac{1}{p(s)} \int_{0}^{1} M(v)\left(2 \epsilon_{2}\left(\|x\|+\left\|x_{0}\right\|\right)+C\right) d v\right) d s \\
\leq 2 \epsilon_{2} R \int_{0}^{1} G(s, s)\left(\frac{1}{p(s)} \int_{0}^{1} M(v) d v\right) d s \\
+\left(C+2 \epsilon_{2}\left\|x_{0}\right\|\right) \\
\times \int_{0}^{1} G(s, s)\left(\frac{1}{p(s)} \int_{0}^{1} M(v) d v\right) d s \\
<R=\|x\| .
\end{gathered}
$$

Hence, $\|T x\|<\|x\|$. By Lemma 11, $i\left(T, K_{R}, K\right)=1$. The proof is completed.

Now, we prove the existence of solutions for boundary value problem (5) by using the Guo-Krasnoselskii fixed point theorem.

Theorem 15. Let $b>(2-\alpha) /(\alpha-1)$, and let $\left(H_{1}\right)$ and $\left(H_{3}\right)$ hold. Then, boundary value problem (5) has at least a positive solution.

Proof. By $\left(H_{3}\right)$, there exists a $Z_{1}>0$ such that

$$
\begin{gathered}
f(\nu, \mu, v) \leq \varepsilon_{1}(\mu+\nu), \\
\mu, v \geq 0, \quad \mu+v \geq Z_{1}, \quad v \in[0,1],
\end{gathered}
$$

where

$$
0<\varepsilon_{1}<\frac{1}{2}\left(\int_{0}^{1} G(s, s)\left(\frac{1}{p(s)} \int_{0}^{1} M(v) d v\right) d s\right)^{-1} .
$$

Taking

$$
C:=\max _{v \in[0,1] 0 \leq \mu, \nu, \mu+\nu \leq Z_{1}}\left|f(\nu, \mu, \nu)-\varepsilon_{1}(\mu+\nu)\right|+1,
$$

we get that

$$
f(v, \mu, \nu) \leq \varepsilon_{1}(\mu+\nu)+C, \quad \mu, v \geq 0, v \in[0,1] .
$$


Assume that

$$
Z_{1} \geq \frac{\left(C+2 \varepsilon_{1}\left\|x_{0}\right\|\right) \int_{0}^{1} G(s, s)\left((1 / p(s)) \int_{0}^{1} M(v) d v\right) d s}{\left(1-2 \varepsilon_{1} \int_{0}^{1} G(s, s)\left((1 / p(s)) \int_{0}^{1} M(v) d v\right) d s\right)} .
$$

By (73), for any $x \in K$ satisfied $\|x\|=Z_{1}$ and $t \in[0,1]$, we have

$$
\begin{aligned}
& |(T x)(t)| \\
& =\int_{0}^{1} G(t, s) \\
& \quad \times\left(\frac{1}{p(s)} \int_{0}^{1} H(s, v)\right. \\
& \times f\left(v,\left(x+x_{0}\right)(v-\tau),\right. \\
& \left.\left.\leq \int_{0}^{1} G(s, s) \quad\left(x+x_{0}\right)(v+\theta)\right) d v\right) d s \\
& \times\left(\frac{1}{p(s)} \int_{0}^{1} M(v)\right. \\
& \leq \int_{0}^{1} G(s, s) \\
& \times\left(\frac{1}{p(s)} \int_{0}^{1} M(v)\right. \\
& \times f\left(v,\left(x+x_{0}\right)(v-\tau),\right. \\
& \left.\left.\left(x+x_{0}\right)(v+\theta)\right) d v\right) d s
\end{aligned}
$$$$
\leq \int_{0}^{1} G(s, s)
$$$$
\times\left(\frac{1}{p(s)} \int_{0}^{1} M(v)\left(2 \varepsilon_{1}\left(\|x\|+\left\|x_{0}\right\|\right)+C\right) d v\right) d s
$$$$
\leq 2 \varepsilon_{1} Z_{1} \int_{0}^{1} G(s, s)\left(\frac{1}{p(s)} \int_{0}^{1} M(v) d v\right) d s
$$$$
+\left(C+2 \varepsilon_{1}\left\|x_{0}\right\|\right) \int_{0}^{1} G(s, s)\left(\frac{1}{p(s)} \int_{0}^{1} M(v) d v\right) d s
$$$$
<Z_{1}
$$$$
=\|x\| \text {. }
$$

Now, if we let

$$
\Omega_{1}=\left\{x \in X:\|x\|<Z_{1}\right\}
$$

then (76) shows that

$$
\|T x\|_{[-\tau, 1+\theta]} \leq\|x\|_{[-\tau, 1+\theta]} \text { for } x \in K \cap \partial \Omega_{1} \text {. }
$$

On the other hand, by $\left(H_{1}\right)$, there is an $r_{0}>0$ such that $0<Z_{2} \leq r_{0}$ implies

$$
\begin{gathered}
f(\nu, \mu, v) \geq L(\mu+v), \\
\mu, v \geq 0, \quad \mu+v \leq 2 Z_{2}, \quad v \in[0,1],
\end{gathered}
$$

where $L>0$ satisfies

$$
\gamma_{1} L \int_{1 / 4}^{3 / 4} G\left(\frac{1}{2}, s\right)\left(\frac{1}{p(s)} \int_{1 / 4}^{3 / 4} \gamma_{2}(v) M(v) d v\right) d s \geq \frac{1}{2} .
$$

Thus for $x \in K$ and $\|x\|=Z_{2}, t \in[0,1]$, we have

$$
\begin{aligned}
& Z_{2}=\|x\| \geq x(v-\tau) \geq \gamma_{1}\|x\| \geq \gamma_{1} Z_{2}, \quad v \in\left[\frac{1}{4}, \frac{3}{4}\right], \\
& Z_{2}=\|x\| \geq x(v+\varphi) \geq \gamma_{1}\|x\| \geq \gamma_{1} Z_{2}, \quad v \in\left[\frac{1}{4}, \frac{3}{4}\right] .
\end{aligned}
$$

In view of (78) and (79), we get that

$$
\begin{aligned}
& \left|(T x)\left(\frac{1}{2}\right)\right| \\
& \geq \int_{1 / 4}^{3 / 4} G\left(\frac{1}{2}, s\right) \\
& \quad \times\left(\frac{1}{p(s)} \int_{1 / 4}^{3 / 4} H(s, v)\right. \\
& \times f\left(v,\left(x+x_{0}\right)(v-\tau),\right. \\
& \geq \int_{1 / 4}^{3 / 4} G\left(\frac{1}{2}, s\right) \\
& \quad \times\left(\frac{1}{p(s)} \int_{1 / 4}^{3 / 4} \gamma_{2}(v) M(v)\right.
\end{aligned}
$$

$$
\times f(v, x(v-\tau), x(v+\theta)) d v) d s
$$




$$
\begin{aligned}
& \geq L \int_{1 / 4}^{3 / 4} G\left(\frac{1}{2}, s\right) \\
& \quad \times\left(\frac{1}{p(s)} \int_{1 / 4}^{3 / 4} \gamma_{2}(v) M(v)\right. \\
& \quad \times(x(v-\tau)+x(v+\theta)) d v) d s
\end{aligned}
$$$$
\geq 2 \gamma_{1} Z_{2} L
$$$$
\times \int_{1 / 4}^{3 / 4} G\left(\frac{1}{2}, s\right)\left(\frac{1}{p(s)} \int_{1 / 4}^{3 / 4} \gamma_{2}(v) M(v) d v\right) d s
$$$$
\geq Z_{2}=\|x\| \text {. }
$$

Now, if we let

$$
\Omega_{2}=\left\{x \in X:\|x\|<Z_{2}\right\}
$$

then (81) shows that

$$
\|T x\|_{[-\tau, 1+\theta]} \geq\|x\|_{[-\tau, 1+\theta]} \quad \text { for } x \in K \cap \partial \Omega_{2} .
$$

Thus, by the first part of Lemma 10, $T$ has a fixed point $x \in K \cap\left(\bar{\Omega}_{2} \backslash \Omega_{1}\right)$ with $Z_{1} \leq\|x\|_{[-\tau, 1+\theta]} \leq Z_{2}$, and accordingly, $x$ is a positive solution of problem (5). The proof is completed.

Theorem 16. Let $b>(2-\alpha) /(\alpha-1)$, and let $\left(H_{2}\right)$ and $\left(H_{3}\right)$ hold. Then, boundary value problem (5) has at least a positive solution.

Proof. By $\left(\mathrm{H}_{2}\right)$ for any $L>0$ satisfying (126), there is a $R_{0}>0$ such that

$$
\begin{gathered}
f(\nu, \mu, \nu) \geq L(\mu+v), \\
\mu, v \geq 0, \quad \mu+v \geq R_{0}, \quad v \in[0,1] .
\end{gathered}
$$

Choose

$$
Z_{3}>\max \left\{Z_{1}, \frac{R_{0}}{2 \gamma_{1}}\right\}
$$

Thus for $x \in K,\|x\|=Z_{3}$ and $t \in[0,1]$, we have

$$
\begin{aligned}
& Z_{3}=\|x\| \geq x(v-\tau) \geq \gamma_{1}\|x\| \geq \gamma_{1} Z_{3}>\frac{R_{0}}{2}, \quad v \in\left[\frac{1}{4}, \frac{3}{4}\right], \\
& Z_{3}=\|x\| \geq x(v+\theta) \geq \gamma_{1}\|x\| \geq \gamma_{1} Z_{3}>\frac{R_{0}}{2}, \quad v \in\left[\frac{1}{4}, \frac{3}{4}\right] .
\end{aligned}
$$

In view of (55) and (59), we get that

$$
\begin{aligned}
& \left|(T x)\left(\frac{1}{2}\right)\right| \\
& \geq \int_{1 / 4}^{3 / 4} G\left(\frac{1}{2}, s\right) \\
& \quad \times\left(\frac{1}{p(s)} \int_{1 / 4}^{3 / 4} H(s, v)\right. \\
& \times f\left(v,\left(x+x_{0}\right)(v-\tau),\right. \\
& \left.\left.\quad\left(x+x_{0}\right)(v+\theta)\right) d v\right) d s
\end{aligned}
$$$$
\geq \int_{1 / 4}^{3 / 4} G\left(\frac{1}{2}, s\right)
$$

$$
\begin{aligned}
\times\left(\frac{1}{p(s)} \int_{1 / 4}^{3 / 4} \gamma_{2}(v) M(v)\right. \\
\times f(v, x(v-\tau),
\end{aligned}
$$

$$
\begin{gathered}
x(v+\theta)) d v) d s \\
\geq L \int_{1 / 4}^{3 / 4} G\left(\frac{1}{2}, s\right) \\
\times\left(\frac{1}{p(s)} \int_{1 / 4}^{3 / 4} \gamma_{2}(v) M(v)\right. \\
\geq 2 \gamma_{1} Z_{3} L \int_{1 / 4}^{3 / 4} G\left(\frac{1}{2}, s\right) \\
\times\left(\frac{1}{p(s)} \int_{1 / 4}^{3 / 4} \gamma_{2}(v) M(v) d v\right) d s \\
\left.\left.\geq Z_{3}=\|x\| . \quad x(v+\theta)\right) d v\right) d s
\end{gathered}
$$

Now, if we let

$$
\Omega_{1}=\left\{x \in X:\|x\|<Z_{3}\right\},
$$

then (144) shows that

$$
\|T x\|_{[-\tau, 1+\theta]} \geq\|x\|_{[-\tau, 1+\theta]} \quad \text { for } x \in K \cap \partial \Omega_{1} .
$$

On the other hand, from Theorem 15 , by $\left(H_{3}\right)$, there exists a $Z_{1}>0$ such that

$$
\begin{gathered}
f(\nu, \mu, v) \leq \varepsilon_{2}(\mu+v), \\
\mu, v \geq 0, \quad \mu+v \geq Z_{1}, \quad v \in[0,1],
\end{gathered}
$$

where

$$
0<\varepsilon_{2}<\frac{1}{2}\left(\int_{0}^{1} G(s, s)\left(\frac{1}{p(s)} \int_{0}^{1} M(v) d v\right) d s\right)^{-1} .
$$


Now, if we let

$$
\Omega_{2}=\left\{x \in X:\|x\|<Z_{1}\right\},
$$

then (119) shows that

$$
\|T x\|_{[-\tau, 1+\theta]} \leq\|x\|_{[-\tau, 1+\theta]} \text { for } x \in K \cap \partial \Omega_{2} .
$$

Thus, by the second part of Lemma 10,T has a fixed point $x \in K \cap\left(\bar{\Omega}_{2} \backslash \Omega_{1}\right)$ with $Z_{3} \leq\|x\|_{[-\tau, 1+\theta]} \leq Z_{1}$, and accordingly, $x$ is a positive solution of problem (5). The proof is completed.

Next, we study the existence of solutions for boundary value problem (5) by the fixed point index theorem. For this purpose, we first give the following lemma.

Lemma 17. Let $L_{0}:=\left(\lambda \int_{0}^{1} G(s, s)\left((1 / p(s)) \int_{0}^{1} M(v) d v\right) d s\right)^{-1}$. Assume that

$$
\lim _{u \rightarrow \infty} \max _{t \in[0,1]} \frac{f(t, u)}{u}=\widetilde{L} \in\left[0, L_{0}\right) .
$$

If there exists a function $g:[0,1] \rightarrow \mathbb{R}$ such that

$$
f(t, u) \geq g(t) \quad \text { for } t \in\left[\frac{1}{4}, \frac{3}{4}\right],
$$

Then boundary value problem for the following fractional functional differential equation:

$$
\begin{gathered}
{ }^{C} D^{\beta}\left(p(t){ }^{C} D^{\alpha} u(t)\right)+\lambda f(t, u)=0, \quad t \in(0,1), \\
{ }^{C} D^{\alpha} u(0)={ }^{C} D^{\alpha} u(1)=\left({ }^{C} D^{\alpha} u(0)\right)^{\prime \prime}=0, \\
a u(t)-b u^{\prime}(t)=\eta(t), \quad t \in[-\tau, 0], \\
c u(t)+d u^{\prime}(t)=\xi(t), \quad t \in[1,1+\theta]
\end{gathered}
$$

has at least a positive solution for $\lambda>0$.

Proof. Let $\lambda>0$. We define the operator $T_{\lambda}$ on $K$ by

$$
\left(T_{\lambda} u\right)(t)=\lambda \int_{0}^{1} G(t, s)\left(\frac{1}{p(s)} \int_{0}^{1} H(s, v) f(v, u) d v\right) d s
$$

In view of Lemma 12 , it can be verified that $T_{\lambda}$ is completely continuous. Then, we prove that $T_{\lambda}$ has a fixed point in $K$.

Set $\epsilon_{0}=L_{0}-\widetilde{L}$. From the assumption, for any given $\epsilon_{0}>0$, there exists $u_{0}>0$ such that

$$
f(t, u) \leq\left(\widetilde{L}+\epsilon_{0}\right) u \quad \forall t \in[0,1], u \geq u_{0} .
$$

For $u \in K$ and $\|u\|=r_{1}:=\max \left\{\lambda, \gamma_{1}^{-1} u_{0}\right\}$, we have

$$
\begin{aligned}
\left(T_{\lambda} u\right)(t) & \leq \lambda \int_{0}^{1} G(s, s)\left(\frac{1}{p(s)} \int_{0}^{1} M(v) f(v, u) d v\right) d s \\
& \leq \lambda L_{0} r_{1} \int_{0}^{1} G(s, s)\left(\frac{1}{p(s)} \int_{0}^{1} M(v) d v\right) d s \\
& \leq r_{1}=\|u\| .
\end{aligned}
$$

Now, if we let $\Omega_{1}=\left\{u \in K:\|u\|<r_{1}\right\}$, then it shows that

$$
\left\|\left(T_{\lambda} u\right)(t)\right\| \leq\|u\|, \quad \text { for } u \in K \cap \partial \Omega_{1} .
$$

\section{Denote}

$$
M_{0}:=\int_{1 / 4}^{3 / 4} G(s, s)\left(\frac{1}{p(s)} \int_{1 / 4}^{3 / 4} \gamma_{2}(v) M(v) g(v) d v\right) d s .
$$

For $u \in K$ and $\|u\|=\lambda \gamma_{1} M_{0}$, we have

$$
\begin{aligned}
&\left(T_{\lambda} u\right)(t) \geq \lambda \int_{1 / 4}^{3 / 4} \gamma_{1} G(s, s) \\
& \times\left(\frac{1}{p(s)} \int_{1 / 4}^{3 / 4} \gamma_{2}(v) M(v) g(v) d v\right) d s \\
& \geq \lambda \gamma_{1} M_{0}=\|u\| .
\end{aligned}
$$

Now, if we let $\Omega_{2}=\left\{u \in K:\|u\|<\lambda \gamma_{1} M_{0}\right\}$, then it shows that

$$
\left\|\left(T_{\lambda} u\right)(t)\right\| \geq\|u\|, \quad \text { for } u \in K \cap \partial \Omega_{2}
$$

Thus, by Lemma $10, T_{\lambda}$ has a fixed point $\tilde{u} \in K \cap\left(\bar{\Omega}_{2} \backslash \Omega_{1}\right)$ between $r_{1}$ and $\lambda \gamma_{1} M_{0}$. Accordingly, $\widetilde{u}$ is a positive solution to (96). The proof is completed.

From Lemma 17, we assume that $\lambda_{1}>0$ is an eigenvalue of

$$
-{ }^{C} D^{\beta}\left(p(t)^{C} D^{\alpha} u\right)=\lambda_{1} u
$$

subject to the following conditions:

$$
a u(0)-b u^{\prime}(0)=0, \quad c u(1)+d u^{\prime}(1)=0,
$$

and the corresponding eigenfunction $\varphi_{1}(t)>0$ for $t \in(0,1)$ such that

$$
\left\|\varphi_{1}\right\|_{[0,1]}=\max _{t \in[0,1]}\left|\varphi_{1}(t)\right|=1
$$

For convenience, we give some conditions as follows.

$\left(H_{4}\right) \liminf \operatorname{int}_{\mu \rightarrow 0^{+}} \min _{t \in[0,1]} f(t, \mu, \nu) /(\mu+\nu)>(1 / 2) \lambda_{1}(1+$ $M_{1}$ ), where

$$
M_{1}=\frac{(\tau+\theta)(1-\tau-\theta)\left\|\varphi_{1}^{\prime}\right\|_{[0,1]}+2(\tau+\theta)}{\int_{0}^{1-\tau-\theta} \gamma_{1} \varphi_{1}(t+\tau) d t+\int_{\tau+\theta}^{1} \gamma_{1} \varphi_{1}(t-\theta) d t}
$$

$\left(H_{5}\right) \liminf _{\mu+\nu \rightarrow+\infty} \min _{t \in[0,1]} f(t, \mu, v) /(\mu+\nu)>$ $(1 / 2) \lambda_{1}\left(1+M_{2}\right)$, where

$$
M_{2}=\frac{(\tau(1-\tau)+\theta(1-\theta))\left\|\varphi_{1}^{\prime}\right\|_{[0,1]}+\tau+\theta}{\int_{0}^{1-\tau} \gamma_{1} \varphi_{1}(t+\tau) d t+\int_{\theta}^{1} \gamma_{1} \varphi_{1}(t-\theta) d t} .
$$


$\left(H_{6}\right) \lim \sup _{\mu+\nu \rightarrow+\infty} \max _{t \in[0,1]} f(t, \mu, v) /(\mu+\nu) \quad>$ $(1 / 2) \lambda_{1}(1-m)$, where

$$
\begin{aligned}
m=( & (\tau(1-\tau)+\theta(1-\theta))\left\|\varphi_{1}^{\prime}\right\|_{[0,1]}+\tau+\theta \\
& \left.\quad-\int_{1-\tau}^{1} \gamma_{1} \varphi_{1}(t) d t-\int_{0}^{\theta} \gamma_{1} \varphi_{1}(t) d t\right) \\
\times & \left(\int_{0}^{1-\tau} \gamma_{1} \varphi_{1}(t+\tau) d t\right. \\
& \left.+\int_{\theta}^{1} \gamma_{1} \varphi_{1}(t-\theta) d t+\tau+\theta\right)^{-1}<1 .
\end{aligned}
$$

$\left(H_{7}\right)$ There is a $h_{1}>0$ such that $0 \leq \mu \leq h_{1}+\left\|x_{0}\right\|_{[-\tau, 0]}$, $0 \leq v \leq h_{1}+\left\|x_{0}\right\|_{[1,1+\theta]}$, and $t \in[0,1]$ imply that

$$
f(t, \mu, v)<k h_{1},
$$

where $k=\left(\int_{0}^{1} G(s, s)\left((1 / p(s)) \int_{0}^{1} M(v) d v\right) d s\right)^{-1}$.

$\left(H_{8}\right)$ There is a $h_{2}>0$ such that $\gamma_{1} h_{2} \leq \mu \leq h_{2}, \gamma_{1} h_{2} \leq \nu \leq$ $h_{2}$, and $t \in[0,1]$ imply that

$$
f(t, \mu, v)>\vartheta h_{2},
$$

$$
\begin{aligned}
& \text { where } 9=\quad\left(\int _ { 1 / 4 } ^ { 3 / 4 } G ( ( 1 / 2 ) , s ) \left((1 / p(s)) \int_{1 / 4}^{3 / 4} \gamma_{2}(v)\right.\right. \\
& \cdot M(v) d v) d s)^{-1} .
\end{aligned}
$$

Lemma 18. Let $\left(H_{7}\right)$ hold. Then, $i\left(T, K_{h_{1}}, K\right)=1$.

Proof. Let $x \in \partial K_{h_{1}}$. Then,

$$
\begin{aligned}
0 & \leq\left(x+x_{0}\right)(v-\tau) \leq\|x\|+\left\|x_{0}\right\|_{[-\tau, 1-\tau]} \\
& =h_{1}+\left\|x_{0}\right\|_{[-\tau, 0]}, \quad v \in[0,1], \\
0 & \leq\left(x+x_{0}\right)(v+\theta) \leq\|x\|+\left\|x_{0}\right\|_{[\theta, 1+\theta]} \\
& =h_{1}+\left\|x_{0}\right\|_{[1,1+\theta]}, \quad v \in[0,1] .
\end{aligned}
$$

Thus, for $v \in[0,1]$, we have by $\left(H_{7}\right)$ that

$$
\begin{aligned}
& (T x)(t) \\
& \leq \int_{0}^{1} G(s, s) \\
& \times\left(\frac{1}{p(s)} \int_{0}^{1} M(v)\right. \\
& \times f\left(v,\left(x+x_{0}\right)(v-\tau),\right. \\
& \left.\left.\left(x+x_{0}\right)(v+\theta)\right) d v\right) d s \\
& <k h_{1} \int_{0}^{1} G(s, s)\left(\frac{1}{p(s)} \int_{0}^{1} M(v) d v\right) d s \\
& =h_{1}=\|x\| .
\end{aligned}
$$

Hence, $\|T x\|<\|x\|$. By Lemma 11, we get $i\left(T, K_{h_{1}}, K\right)=1$. The proof is complete.
Lemma 19. Let $\left(H_{8}\right)$ hold. Then, $i\left(T, K_{h_{2}}, K\right)=0$.

Proof. Let $x \in \partial K_{h_{2}}$. Then,

$$
\begin{aligned}
& h_{2}=\|x\| \geq x(v-\tau) \geq \gamma_{1}\|x\| \geq \gamma_{1} h_{2}, \quad v \in\left[\frac{1}{4}, \frac{3}{4}\right], \\
& h_{2}=\|x\| \geq x(v+\theta) \geq \gamma_{1}\|x\| \geq \gamma_{1} h_{2}, \quad v \in\left[\frac{1}{4}, \frac{3}{4}\right] .
\end{aligned}
$$

Thus, for $v \in[0,1]$, we have by $\left(H_{8}\right)$ that

$$
\begin{aligned}
(T x)(t) \geq \int_{1 / 4}^{3 / 4} G\left(\frac{1}{2}, s\right) & \\
& \times\left(\frac{1}{p(s)} \int_{1 / 4}^{3 / 4} H(s, v)\right. \\
& \times f\left(v,\left(x+x_{0}\right)(v-\tau),\right. \\
\geq & \left.\left.\int_{1 / 4}^{3 / 4} G\left(\frac{1}{2}, s\right)\left(x+x_{0}\right)(v+\theta)\right) d v\right) d s \\
& \times\left(\frac{1}{p(s)} \int_{1 / 4}^{3 / 4} \gamma_{2}(v) M(v)\right. \\
> & 9 h_{2} \int_{1 / 4}^{3 / 4} G\left(\frac{1}{2}, s\right)\left(\frac{1}{p(s)} \int_{1 / 4}^{3 / 4} \gamma_{2}(v) M(v) d v\right) d s \\
= & h_{2}=\|x\| .
\end{aligned}
$$

Hence, $\|T x\|>\|x\|$. By Lemma 11, we get $i\left(T, K_{h_{2}}, K\right)=0$. The proof is complete.

Theorem 20. Let $\left(H_{4}\right),\left(H_{5}\right)$, and $\left(H_{7}\right)$ hold. Then, boundary value problem (5) has at least two positive solutions $u_{1}$ and $u_{2}$ such that

$$
0<\left\|u_{1}\right\|_{[0,1]}<h_{1}<\left\|u_{2}\right\|_{[0,1]} .
$$

Proof. According to Lemma 18, we get that $i\left(T, K_{h_{1}}, K\right)=1$. Let $0<m<1<n$ and $f_{1}(\nu, \mu, \nu)=(\mu+\nu)^{m}+(\mu+\nu)^{n}$, for $\mu, \nu \geq 0$. Then, $f_{1}(v, \mu, \nu)$ satisfy $\left(H_{1}\right)$ and $\left(H_{2}\right)$. 
Define $T_{1}: K \rightarrow K$ by

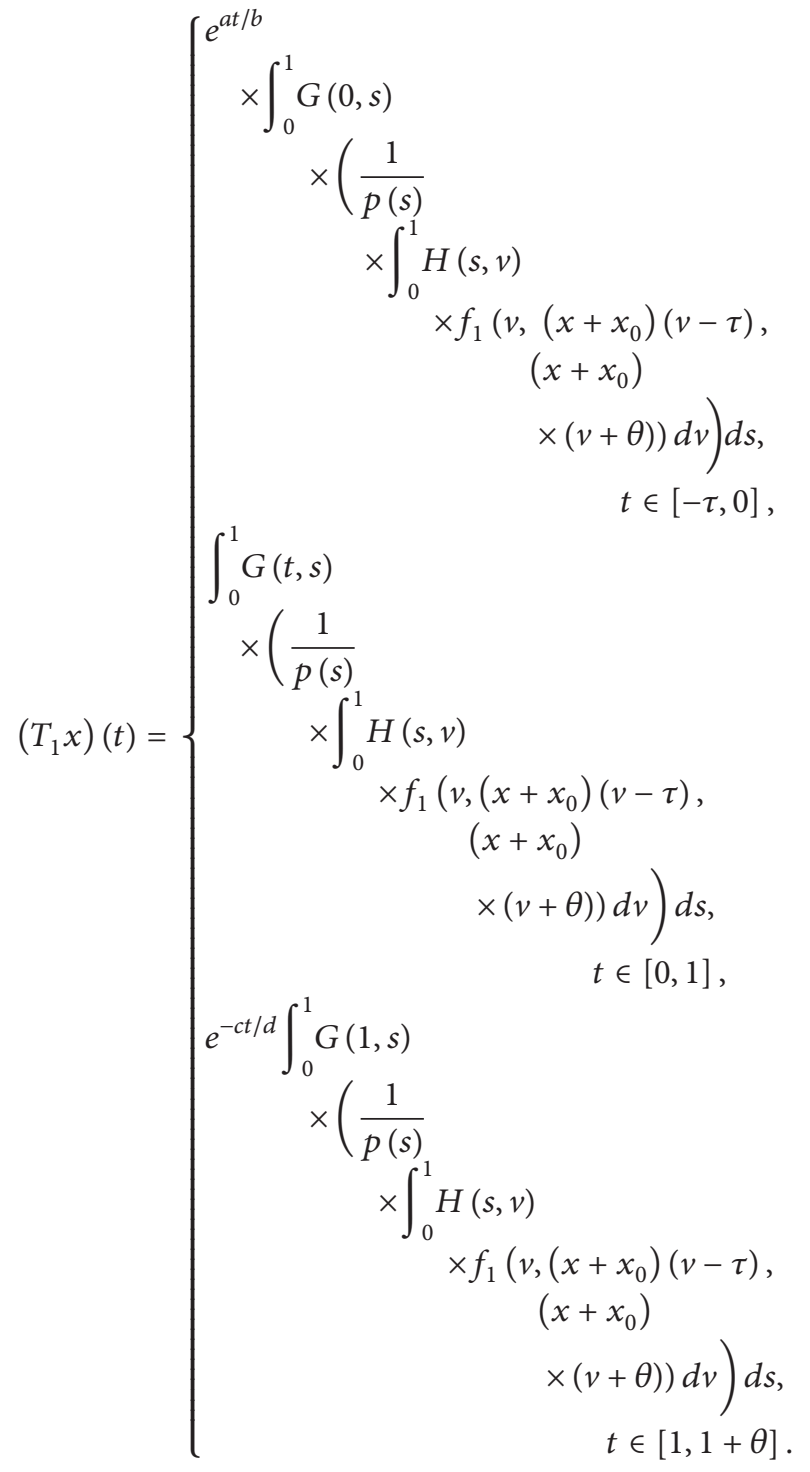

It is easy to know that $T_{1}$ is a completely continuous operator.

Also, according to Lemma 13, we have that there exist $0<$ $r_{0}<R_{0}<\infty$, such that

$$
\begin{gathered}
i\left(T_{1}, K_{r}, K\right)=0, \quad 0<r \leq r_{0}, \\
i\left(T_{1}, K_{R}, K\right)=0, \quad R \geq R_{0} .
\end{gathered}
$$

Define $A:[0,1] \times K \rightarrow K$ by $A(s, x)=(1-s) T x+s T_{1} x$. For any $s_{1}, s_{2} \in[0,1], \omega>0$, we have

$$
\begin{aligned}
& \left|A\left(s_{1}, x\right)-A\left(s_{2}, x\right)\right| \\
& \quad \leq\left|\left(1-s_{1}\right) T x+s_{1} T_{1} x-\left(1-s_{2}\right) T x-s_{2} T_{1} x\right| \\
& \quad \leq\left|s_{1}-s_{2}\right|\left(\left\|T_{1} x\right\|+\|T x\|\right) .
\end{aligned}
$$

Note that $\left\|T_{1} x\right\|+\|T x\|$ is uniformly bounded in $K_{\omega}$. Thus, $A(s, x)$ is continuous on $x \in K_{\omega}$ uniformly for $s \in[0,1]$.
Then, we conclude that $A$ is a completely continuous operator on $[0,1] \times K_{\omega}$.

By $\left(H_{4}\right)$ and definition of $f_{1}$, there exists a $\epsilon_{3}>0$ and $0<r_{1} \leq r_{0}$ such that

$$
\begin{array}{r}
f(t, \mu, \nu) \geq \frac{1}{2}\left(\lambda_{1}\left(1+M_{1}\right)+\epsilon_{3}\right)(\mu+\nu), \\
\mu, \nu \geq 0, \mu+\nu \leq 2 r_{1}, \quad t \in[0,1], \\
f_{1}(t, \mu, v) \geq \frac{1}{2}\left(\lambda_{1}\left(1+M_{1}\right)+\epsilon_{3}\right)(\mu+\nu), \\
\mu, \nu \geq 0, \quad \mu+v \leq 2 r_{1}, \quad t \in[0,1] .
\end{array}
$$
$\partial K_{r_{1}}$.

Next, we prove that $A(s, x) \neq x$ for all $s \in[0,1]$ and $x \in$

In fact, if there exist $s_{1} \in[0,1]$ and $x_{1} \in \partial K_{r_{1}}$ such that $A\left(s_{1}, x_{1}\right)=x_{1}$, then $x_{1}(t)$ satisfies the following:

$$
\begin{aligned}
-{ }^{C} D^{\beta}\left(p(t){ }^{C} D^{\alpha} x_{1}(t)\right) & \\
= & \left(1-s_{1}\right) f\left(t,\left(x_{1}+x_{0}\right)(t-\tau),\left(x_{1}+x_{0}\right)(t+\theta)\right) \\
& +s_{1} f_{1}\left(t,\left(x_{1}+x_{0}\right)(t-\tau),\left(x_{1}+x_{0}\right)(t+\theta)\right)
\end{aligned}
$$

and the following boundary conditions:

$$
\begin{gathered}
a x_{1}(t)-b x_{1}^{\prime}(t)=0, \quad t \in[-\tau, 0], \\
c x_{1}(t)+d x_{1}^{\prime}(t)=0, \quad t \in[1,1+\theta] .
\end{gathered}
$$

Multiplying both sides of (122) by $\varphi_{1}(t)$, then integrating from 0 to 1 and using Green's formula, we obtain

$$
\begin{aligned}
& \lambda_{1} \int_{0}^{1} x_{1}(t) \varphi_{1}(t) d t \\
& =\int_{0}^{1}\left(-{ }^{C} D^{\beta}\left(p(t)^{C} D^{\alpha} x_{1}(t)\right)\right) \varphi_{1}(t) d t \\
& =\left(1-s_{1}\right) \\
& \quad \times \int_{0}^{1} f\left(t,\left(x_{1}+x_{0}\right)(t-\tau),\left(x_{1}+x_{0}\right)(t+\theta)\right) \varphi_{1}(t) d t \\
& \quad+s_{1} \int_{0}^{1} f_{1}\left(t,\left(x_{1}+x_{0}\right)(t-\tau),\left(x_{1}+x_{0}\right)(t+\theta)\right) \varphi_{1}(t) d t .
\end{aligned}
$$


Thus, we know that

$$
\begin{aligned}
& \lambda_{1} \int_{0}^{1} x_{1}(t) \varphi_{1}(t) d t \\
& \geq\left(1-s_{1}\right) \\
& \times \int_{\tau}^{1-\theta} f\left(t,\left(x_{1}+x_{0}\right)(t-\tau),\left(x_{1}+x_{0}\right)(t+\theta)\right) \\
& \times \varphi_{1}(t) d t \\
& +s_{1} \int_{\tau}^{1-\theta} f_{1}\left(t,\left(x_{1}+x_{0}\right)(t-\tau),\left(x_{1}+x_{0}\right)(t+\theta)\right) \\
& \times \varphi_{1}(t) d t \\
& =\left(1-s_{1}\right) \int_{\tau}^{1-\theta} f\left(t, x_{1}(t-\tau), x_{1}(t+\theta)\right) \varphi_{1}(t) d t \\
& +s_{1} \int_{\tau}^{1-\theta} f_{1}\left(t, x_{1}(t-\tau), x_{1}(t+\theta)\right) \varphi_{1}(t) d t \\
& \geq \frac{1}{2}\left(1-s_{1}\right)\left(\lambda_{1}\left(1+M_{1}\right)+\epsilon_{3}\right) \\
& \times \int_{\tau}^{1-\theta}\left(x_{1}(t-\tau)+x_{1}(t+\theta)\right) \varphi_{1}(t) d t \\
& +\frac{1}{2} s_{1}\left(\lambda_{1}\left(1+M_{1}\right)+\epsilon_{3}\right) \\
& \times \int_{\tau}^{1-\theta}\left(x_{1}(t-\tau)+x_{1}(t+\theta)\right) \varphi_{1}(t) d t \\
& =\frac{1}{2}\left(\lambda_{1}\left(1+M_{1}\right)+\epsilon_{3}\right) \\
& \times\left(\int_{\tau}^{1-\theta} x_{1}(t-\tau) \varphi_{1}(t) d t\right. \\
& \left.+\int_{\tau}^{1-\theta} x_{1}(t+\theta) \varphi_{1}(t) d t\right) \\
& =\frac{1}{2}\left(\lambda_{1}\left(1+M_{1}\right)+\epsilon_{3}\right) \\
& \times\left(\int_{0}^{1-\theta-\tau} x_{1}(t) \varphi_{1}(t+\tau) d t\right. \\
& \left.+\int_{\tau+\theta}^{1} x_{1}(t) \varphi_{1}(t-\theta) d t\right) .
\end{aligned}
$$

Then, we have

$$
\begin{aligned}
& \left(\lambda_{1} M_{1}+\epsilon_{3}\right) \\
& \quad \times\left(\int_{0}^{1-\theta-\tau} x_{1}(t) \varphi_{1}(t+\tau) d t\right. \\
& \left.\quad+\int_{\tau+\theta}^{1} x_{1}(t) \varphi_{1}(t-\theta) d t\right)
\end{aligned}
$$

$$
\begin{aligned}
\leq & 2 \lambda_{1} \int_{0}^{1} x_{1}(t) \varphi_{1}(t) d t \\
& -\lambda_{1}\left(\int_{0}^{1-\theta-\tau} x_{1}(t) \varphi_{1}(t+\tau) d t\right. \\
& \left.+\int_{\tau+\theta}^{1} x_{1}(t) \varphi_{1}(t-\theta) d t\right) \\
= & \lambda_{1} \int_{0}^{1-\theta-\tau} x_{1}(t)\left(\varphi_{1}(t)-\varphi_{1}(t+\tau)\right) d t \\
& +\lambda_{1} \int_{1-\tau-\theta}^{1} x_{1}(t) \varphi_{1}(t) d t \\
& +\lambda_{1} \int_{\tau+\theta}^{1} x_{1}(t)\left(\varphi_{1}(t)-\varphi_{1}(t-\theta)\right) d t \\
& +\lambda_{1} \int_{0}^{\tau+\theta} x_{1}(t) \varphi_{1}(t) d t \\
& +\lambda_{1}(\theta+\tau)\left\|\varphi_{1}\right\|_{[0,1]}\left\|x_{1}\right\| \\
& +\lambda_{1}(\theta+\tau)\left\|\varphi_{1}\right\|_{[0,1]}\left\|x_{1}\right\| \\
& +\lambda_{1} \theta(1-\theta-\tau)\left\|\varphi_{1}^{\prime}\right\|_{[0,1]}\left\|x_{1}\right\| \\
& +x_{1} \|
\end{aligned}
$$

By the definition of $K$, we have

$$
\begin{aligned}
& \int_{0}^{1-\theta-\tau} x_{1}(t) \varphi_{1}(t+\tau) d t+\int_{\tau+\theta}^{1} x_{1}(t) \varphi_{1}(t-\theta) d t \\
& \quad \geq \gamma_{1}\left\|x_{1}\right\|\left(\int_{0}^{1-\theta-\tau} \varphi_{1}(t+\tau) d t+\int_{\theta+\tau}^{1} \varphi_{1}(t-\theta) d t\right) .
\end{aligned}
$$

Combining with (126), we get that

$$
\begin{aligned}
& \left(\lambda_{1} M_{1}+\epsilon_{3}\right) \gamma_{1}\left(\int_{0}^{1-\theta-\tau} \varphi_{1}(t+\tau) d t+\int_{\theta+\tau}^{1} \varphi_{1}(t-\theta) d t\right) \\
& \leq \lambda_{1}\left((\theta+\tau)(1-\theta-\tau)\left\|\varphi_{1}^{\prime}\right\|_{[0,1]}+2(\theta+\tau)\right)
\end{aligned}
$$

which is a contradiction for definition of $M_{1}$ in $\left(H_{4}\right)$. Thus, $A(s, x) \neq x$ for $x \in \partial K_{r}$ and $s \in[0,1]$. In view of (118) and homotopy invariance of the fixed point index, we obtain

$$
\begin{aligned}
i\left(T, K_{r_{1}}, K\right) & =i\left(H(0, \cdot), K_{r_{1}}, K\right) \\
& =i\left(H(1, \cdot), K_{r_{1}}, K\right)=i\left(T_{1}, K_{r_{1}}, K\right)=0 .
\end{aligned}
$$


On the other hand, by $\left(H_{5}\right)$ and definition of $f_{1}$, there are $\epsilon_{4}>0$ and $R_{1}>h_{1}$ such that

$$
\begin{aligned}
f(t, \mu, \nu) & \geq \frac{1}{2}\left(\lambda_{1}\left(1+M_{2}\right)+\epsilon_{4}\right)(\mu+\nu), \\
\mu, v & \geq 0, \quad \mu+v>R^{\prime}, \quad t \in[0,1], \\
f_{1}(t, \mu, \nu) & \geq \frac{1}{2}\left(\lambda_{1}\left(1+M_{2}\right)+\epsilon_{4}\right)(\mu+v), \\
\mu, v & \geq 0, \quad \mu+v>R^{\prime}, \quad t \in[0,1] .
\end{aligned}
$$

Setting

$$
\begin{aligned}
C:= & \max _{t \in[0,1] 0 \leq \mu, \nu, \mu+\nu \leq R^{\prime}} \max _{+}\left|f(t, \mu, \nu)-\left(\lambda_{1}\left(1+M_{2}\right)+\epsilon_{4}\right)(\mu+\nu)\right| \\
+\max _{t \in[0,1] 0 \leq \mu, \nu, \mu+\nu \leq R^{\prime}} \max _{0} \mid & f_{1}(t, \mu, \nu) \\
& -\left(\lambda_{1}\left(1+M_{2}\right)+\epsilon_{4}\right)(\mu+\nu) \mid+1,
\end{aligned}
$$

and it follows that

$$
\begin{array}{r}
f(t, \mu, \nu) \geq \frac{1}{2}\left(\lambda_{1}\left(1+M_{2}\right)+\epsilon_{4}\right)(\mu+\nu)+C, \\
\mu, \nu \geq 0, \quad t \in[0,1], \\
f_{1}(t, \mu, \nu) \geq \frac{1}{2}\left(\lambda_{1}\left(1+M_{2}\right)+\epsilon_{4}\right)(\mu+\nu)+C, \\
\mu, \nu \geq 0, \quad t \in[0,1] .
\end{array}
$$

Then, we prove that there exists a $R_{1} \geq R^{\prime}$ such that $A(s, x) \neq x$ for all $s \in[0,1]$ and $x \in K,\|x\| \geq R_{1}$. In fact, if there exist $s_{1} \in[0,1]$ and $x_{1} \in K$ such that $A\left(s_{1}, x_{1}\right)=x_{1}$, we get that

$$
\begin{aligned}
& \lambda_{1} \int_{0}^{1} x_{1}(t) \varphi_{1}(t) d t \\
& \geq \frac{1}{2}\left(1-s_{1}\right)\left(\lambda_{1}\left(1+M_{2}\right)+\epsilon_{4}\right) \\
& \quad \times \int_{0}^{1}\left(\left(x_{1}+x_{0}\right)(t-\tau)+\left(x_{1}+x_{0}\right)(t+\theta)\right) \\
& \quad \times \varphi_{1}(t) d t-\left(1-s_{1}\right) C \int_{0}^{1} \varphi_{1}(t) d t \\
& \quad+\frac{1}{2} s_{1}\left(\lambda_{1}\left(1+M_{2}\right)+\epsilon_{4}\right) \\
& \quad \times \int_{0}^{1}\left(\left(x_{1}+x_{0}\right)(t-\tau)+\left(x_{1}+x_{0}\right)(t+\theta)\right) \\
& \quad \times \varphi_{1}(t) d t-s_{1} C \int_{0}^{1} \varphi_{1}(t) d t \\
& \quad \frac{1}{2}\left(\lambda_{1}\left(1+M_{2}\right)+\epsilon_{4}\right) \\
& \quad \times \int_{0}^{1}\left(x_{1}(t-\tau)+x_{1}(t+\theta)\right) \varphi_{1}(t) d t
\end{aligned}
$$

$$
\begin{aligned}
\geq & \frac{1}{2}\left(\lambda_{1}\left(1+M_{2}\right)+\epsilon_{4}\right) \\
& \times\left(\int_{\tau}^{1} x_{1}(t-\tau) \varphi_{1}(t) d t+\int_{0}^{1-\theta} x_{1}(t+\theta) \varphi_{1}(t) d t\right) \\
& +C \int_{0}^{1} \varphi_{1}(t) d t \\
= & \frac{1}{2}\left(\lambda_{1}\left(1+M_{2}\right)+\epsilon_{4}\right) \\
& \times\left(\int_{0}^{1-\tau} x_{1}(t) \varphi_{1}(t+\tau) d t+\int_{\theta}^{1} x_{1}(t) \varphi_{1}(t-\theta) d t\right) \\
& +C \int_{0}^{1} \varphi_{1}(t) d t .
\end{aligned}
$$

Then, we have

$$
\begin{aligned}
& \left(\lambda_{1} M_{2}+\epsilon_{4}\right) \\
& \quad \times\left(\int_{0}^{1-\tau} x_{1}(t) \varphi_{1}(t+\tau) d t+\int_{\theta}^{1} x_{1}(t) \varphi_{1}(t-\theta) d t\right) \\
& \leq \lambda_{1} \int_{0}^{1-\tau} x_{1}(t)\left(\varphi_{1}(t)-\varphi_{1}(t+\tau)\right) d t \\
& \quad+\lambda_{1} \int_{1-\tau}^{1} x_{1}(t) \varphi_{1}(t) d t \\
& \quad+\lambda_{1} \int_{\theta}^{1} x_{1}(t)\left(\varphi_{1}(t)-\varphi_{1}(t-\theta)\right) d t \\
& \quad+\lambda_{1} \int_{0}^{\theta} x_{1}(t) \varphi_{1}(t) d t+2 C \int_{0}^{1} \varphi_{1}(t) d t \\
& \leq \lambda_{1} \tau(1-\tau)\left\|\varphi_{1}^{\prime}\right\|_{[0,1]}\left\|x_{1}\right\|+\lambda_{1} \tau\left\|_{1}\right\|_{[0,1]}\left\|x_{1}\right\| \\
& \quad+\lambda_{1} \theta(1-\theta)\left\|\varphi_{1}^{\prime}\right\|_{[0,1]}\left\|x_{1}\right\| \\
& +\lambda_{1} \theta\left\|\varphi_{1}\right\|_{[0,1]}\left\|x_{1}\right\|+2 C\left\|\varphi_{1}\right\|_{[0,1]} \\
& =\lambda_{1}\left(\tau(1-\tau)+\theta(1-\theta)\left\|\varphi_{1}^{\prime}\right\|_{[0,1]}+\theta+\tau\right) \\
& \quad \times\left\|x_{1}\right\|+2 C .
\end{aligned}
$$

By the definition of $K$, we get that

$$
\begin{aligned}
& \int_{0}^{1-\tau} x_{1}(t) \varphi_{1}(t+\tau) d t+\int_{\theta}^{1} x_{1}(t) \varphi_{1}(t-\theta) d t \\
& \quad \geq \gamma_{1}\left\|x_{1}\right\|\left(\int_{0}^{1-\tau} \varphi_{1}(t+\tau) d t+\int_{\theta}^{1} \varphi_{1}(t-\theta) d t\right) .
\end{aligned}
$$


Combining with (134), we conclude that

$$
\begin{array}{r}
\left(\lambda_{1} M_{2}+\epsilon_{4}\right) \gamma_{1}\left\|x_{1}\right\|\left(\int_{0}^{1-\tau} \varphi_{1}(t+\tau) d t+\int_{\theta}^{1} \varphi_{1}(t-\theta) d t\right) \\
\leq \lambda_{1}\left((\tau(1-\tau)+\theta(1-\theta))\left\|\varphi_{1}^{\prime}\right\|_{[0,1]}+\theta+\tau\right)\left\|x_{1}\right\|+2 C
\end{array}
$$

that is,

$$
\left\|x_{1}\right\| \leq \frac{2 C}{\epsilon_{4} \gamma_{1}\left(\int_{0}^{1-\tau} \varphi_{1}(t+\tau) d t+\int_{\theta}^{1} \varphi_{1}(t-\theta) d t\right)}=\bar{R}
$$

Let $R_{1}=1+\max \left\{R^{\prime}, \bar{R}\right\}$. Then, we obtain $A(s, x) \neq x$ for $x \in$ $\partial K_{r}$ and $s \in[0,1]$. In view of (118) and homotopy invariance of the fixed point index, we obtain

$$
\begin{aligned}
i\left(T, K_{R_{1}}, K\right) & =i\left(A(0, \cdot), K_{R_{1}}, K\right)=i\left(A(1, \cdot), K_{R_{1}}, K\right) \\
& =i\left(T_{1}, K_{R_{1}}, K\right)=0 .
\end{aligned}
$$

By using additivity, we get that

$$
i\left(T, K_{R_{1}} \backslash \bar{K}_{h_{1}}, K\right)=-1, \quad i\left(T, K_{h_{1}} \backslash \bar{K}_{r_{1}}, K\right)=1 .
$$

Since

$$
\begin{aligned}
\left\|u_{i}\right\|_{[0,1]} & =\left\|x_{i}+x_{0}\right\|_{[0,1]} \\
& =\left\|x_{i}\right\|_{[0,1]}=\left\|T x_{i}\right\|_{[0,1]} \\
& =\left\|T x_{i}\right\|=\left\|x_{i}\right\|,
\end{aligned}
$$

it follows that $u_{1}(t)$ and $u_{2}(t)$ satisfy

$$
0<\left\|u_{1}\right\|_{[0,1]}=\left\|x_{1}\right\|<h_{1}<\left\|x_{2}\right\|=\left\|u_{2}\right\|_{[0,1]} .
$$

The proof is completed.

Theorem 21. Let $\left(H_{6}\right),\left(H_{7}\right)$, and $\left(H_{8}\right)$ hold. Assume that $0<$ $h_{1}<h_{2}$; then boundary value problem (5) has at least two positive solutions $u_{1}$ and $u_{2}$ such that

$$
h_{1}<\left\|u_{1}\right\|_{[0,1]}<h_{2}<\left\|u_{2}\right\|_{[0,1]} .
$$

Proof. According to Lemmas 18 and 19, we obtain

$$
\begin{aligned}
& i\left(T, K_{h_{1}}, K\right)=1, \\
& i\left(T, K_{h_{2}}, K\right)=0 .
\end{aligned}
$$

By $\left(H_{6}\right)$ and definition of $f_{1}$, there are $\epsilon_{5}>0$ and $R_{2}>h_{2}$ such that

$$
\begin{gathered}
f(t, \mu, \nu) \leq \frac{1}{2}\left(\lambda_{1}\left(1-M_{3}\right)-\epsilon_{5}\right)(\mu+\nu), \\
\mu, \nu \geq 0, \quad \mu+\nu>R^{\prime}, \quad t \in[0,1] .
\end{gathered}
$$

Let

$$
\begin{aligned}
C:=\max _{t \in[0,1]} \max _{0 \leq \mu, \nu, \mu+\nu \leq R^{\prime}} \mid & f(t, \mu, \nu) \\
& -\left(\lambda_{1}\left(1-M_{3}\right)-\epsilon_{5}\right)(\mu+\nu) \mid+1,
\end{aligned}
$$

and it follows that

$$
\begin{array}{r}
f(t, \mu, \nu) \leq \frac{1}{2}\left(\lambda_{1}\left(1-M_{3}\right)-\epsilon_{5}\right)(\mu+\nu)+C, \\
\mu, \nu \geq 0, \quad t \in[0,1] .
\end{array}
$$

Define $A:[0,1] \times K \rightarrow K$ by $A(s, x)=s T x$. For any $s_{1}, s_{2} \in[0,1], \omega>0$, we have

$$
\left|A\left(s_{1}, x\right)-A\left(s_{2}, x\right)\right| \leq\left|s_{1}-s_{2}\right|\|T x\|
$$

Thus, $A(s, x)$ is continuous on $x \in K_{\omega}$ uniformly for $s \in[0,1]$. Then, we conclude that $A$ is also a completely continuous operator on $[0,1] \times K_{\omega}$.

Next, we prove that there exists a $R_{1} \geq R^{\prime}$ such that $A(s, x) \neq x$ for all $s \in[0,1]$ and $x \in K,\|x\| \geq R_{1}$.

In fact, if there exist $s_{2} \in[0,1]$ and $x_{2} \in K$ such that $A\left(s_{2}, x_{2}\right)=x_{2}$, then $x_{2}(t)$ satisfies the following:

$$
\begin{aligned}
{ }^{-}{ }^{C} D^{\beta}\left(p(t){ }^{C} D^{\alpha} x_{2}(t)\right) & \\
= & s_{2} f_{1}\left(t,\left(x_{2}+x_{0}\right)(t-\tau),\right. \\
& \left.\quad\left(x_{2}+x_{0}\right)(t+\theta)\right), \quad t \in(0,1) .
\end{aligned}
$$

Multiplying both sides of (149) by $\varphi_{1}(t)$ then integrating from 0 to 1 and using Green's formula, we get that

$$
\begin{aligned}
& \lambda_{1} \int_{0}^{1} x_{2}(t) \varphi_{1}(t) d t \\
& =\int_{0}^{1}\left(-{ }^{C} D^{\beta}\left(p(t)^{C} D^{\alpha} x_{2}(t)\right)\right) \varphi_{1}(t) d t \\
& =s_{2} \int_{0}^{1} f_{1}\left(t,\left(x_{2}+x_{0}\right)(t-\tau),\left(x_{2}+x_{0}\right)(t+\theta)\right) \varphi_{1}(t) d t .
\end{aligned}
$$

Thus, we get that

$$
\begin{aligned}
& \lambda_{1} \int_{0}^{1} x_{1}(t) \varphi_{1}(t) d t \\
& =s_{1} \int_{0}^{1} f\left(t,\left(x_{1}+x_{0}\right)(t-\tau),\right. \\
& \left.\left(x_{1}+x_{0}\right)(t+\theta)\right) \varphi_{1}(t) d t
\end{aligned}
$$




$$
\begin{array}{cc}
\leq \frac{1}{2}\left(\lambda_{1}\left(1-M_{3}\right)-\epsilon_{5}\right) & \times\left((\tau+\theta)\left(\left\|x_{1}\right\|+\left\|x_{0}\right\|\right)+\int_{0}^{1-\tau} x_{1}(t) \varphi_{1}(t+\tau) d t\right. \\
\times \int_{0}^{1}\left(\left(x_{1}+x_{0}\right)(t-\tau)\right. & \left.+\int_{\theta}^{1} x_{1}(t) \varphi_{1}(t-\theta) d t+\left\|\varphi_{1}\right\|_{[0,1]}\right)+C . \\
\left.\quad+\left(x_{1}+x_{0}\right)(t+\theta)\right) \varphi_{1}(t) d t &
\end{array}
$$$$
+C \int_{0}^{1} \varphi_{1}(t) d t
$$$$
=\frac{1}{2}\left(\lambda_{1}\left(1-M_{3}\right)-\epsilon_{5}\right)
$$$$
\times\left(\int_{0}^{\tau}\left(x_{1}+x_{0}\right)(t-\tau) \varphi_{1}(t) d t\right.
$$$$
+\int_{\tau}^{1}\left(x_{1}+x_{0}\right)(t-\tau) \varphi_{1}(t) d t
$$$$
+\int_{0}^{1-\theta}\left(x_{1}+x_{0}\right)(t+\theta) \varphi_{1}(t) d t
$$$$
\left.+\int_{1-\theta}^{1}\left(x_{1}+x_{0}\right)(t+\theta) \varphi_{1}(t) d t\right)
$$$$
+C \int_{0}^{1} \varphi_{1}(t) d t
$$$$
=\frac{1}{2}\left(\lambda_{1}\left(1-M_{3}\right)-\epsilon_{5}\right)
$$$$
\times\left(\int_{0}^{\tau}\left(x_{1}+x_{0}\right)(t-\tau) \varphi_{1}(t) d t\right.
$$$$
+\int_{0}^{1-\tau} x_{1}(t) \varphi_{1}(t+\tau) d t
$$$$
+\int_{\theta}^{1} x_{1}(t) \varphi_{1}(t-\theta) d t
$$$$
\left.+\int_{1-\theta}^{1}\left(x_{1}+x_{0}\right)(t+\theta) \varphi_{1}(t) d t\right)
$$$$
+C \int_{0}^{1} \varphi_{1}(t) d t
$$$$
\leq \frac{1}{2}\left(\lambda_{1}\left(1-M_{3}\right)-\epsilon_{5}\right)
$$$$
\times\left(\tau\left\|x_{1}+x_{0}\right\|\left\|\varphi_{1}\right\|_{[0,1]}\right.
$$$$
+\int_{0}^{1-\tau} x_{1}(t) \varphi_{1}(t+\tau) d t
$$$$
\left.+\int_{\theta}^{1} x_{1}(t) \varphi_{1}(t-\theta) d t+\theta\left\|x_{1}+x_{0}\right\|\left\|\varphi_{1}\right\|_{[0,1]}\right)
$$

$+C\left\|\varphi_{1}\right\|_{[0,1]}$

$\leq \frac{1}{2}\left(\lambda_{1}\left(1-M_{3}\right)-\epsilon_{5}\right)$

$$
\begin{aligned}
& \left(\lambda_{1} M_{3}+\epsilon_{5}\right) \\
& \quad \times\left(\int_{0}^{1-\tau} x_{1}(t) \varphi_{1}(t+\tau) d t\right. \\
& \left.\quad+\int_{\theta}^{1} x_{1}(t) \varphi_{1}(t-\theta) d t+(\tau+\theta)\left(\left\|x_{1}\right\|+\left\|x_{0}\right\|\right)\right) \\
& \leq \lambda_{1} \int_{0}^{1-\tau} x_{1}(t)\left(\varphi_{1}(t+\tau)-\varphi_{1}(t)\right) d t \\
& \quad+\lambda_{1} \int_{\theta}^{1} x_{1}(t)\left(\varphi_{1}(t-\theta)-\varphi_{1}(t)\right) d t \\
& \quad+\lambda_{1}(\theta+\tau)\left(\left\|x_{1}\right\|+\left\|x_{0}\right\|\right) \\
& \quad-\lambda_{1} \int_{1-\tau}^{1} x_{1}(t) \varphi_{1}(t) d t-\lambda_{1} \int_{0}^{\theta} x_{1}(t) \varphi_{1}(t) d t+2 C \\
& \leq \lambda_{1} \tau(1-\tau)\left\|\varphi_{1}^{\prime}\right\|_{[0,1]}\left\|x_{1}\right\|+\lambda_{1} \theta(1-\theta)\left\|\varphi_{1}^{\prime}\right\|_{[0,1]}\left\|x_{1}\right\| \\
& -\lambda_{1} \gamma_{1} \int_{1-\tau}^{1} \varphi_{1}(t) d t\left\|x_{1}\right\| \\
& +\lambda_{1}(\theta+\tau)\left(\left\|x_{1}\right\|+\left\|x_{0}\right\|\right) \\
& -\lambda_{1} \gamma_{1} \int_{0}^{\theta} \varphi_{1}(t) d t\left\|x_{1}\right\|+2 C .
\end{aligned}
$$

By the definition of $K$, we get that

$$
\begin{aligned}
& \int_{0}^{1-\tau} x_{1}(t) \varphi_{1}(t+\tau) d t+\int_{\theta}^{1} x_{1}(t) \varphi_{1}(t-\theta) d t \\
& \quad \geq \gamma_{1}\left\|x_{1}\right\|\left(\int_{0}^{1-\tau} \varphi_{1}(t+\tau) d t+\int_{\theta}^{1} \varphi_{1}(t-\theta) d t\right) .
\end{aligned}
$$

This combined with (126) gives,

$$
\begin{aligned}
& \left(\lambda_{1} M_{3}+\epsilon_{5}\right) \\
& \quad \times \gamma_{1}\left(\int_{0}^{1-\tau} \varphi_{1}(t+\tau) d t\right. \\
& \left.\quad+\int_{\theta}^{1} \varphi_{1}(t-\theta) d t+\tau+\theta\right)\left\|x_{1}\right\|
\end{aligned}
$$




$$
\begin{aligned}
\leq \lambda_{1}\left(\tau(1-\tau)\left\|\varphi_{1}^{\prime}\right\|_{[0,1]}+\theta(1-\theta)\left\|\varphi_{1}^{\prime}\right\|_{[0,1]}+\theta+\tau\right. \\
\quad-\int_{1-\tau}^{1} \varphi_{1}(t) d t \\
\left.\quad-\int_{1}^{\theta} \varphi_{1}(t) d t\right)\left\|x_{1}\right\|+2 C
\end{aligned}
$$

that is,

$$
\begin{aligned}
& \left\|x_{1}\right\| \\
& \leq \frac{2 C+\lambda_{1}(\theta+\tau)\left\|x_{0}\right\|}{\epsilon_{5} \gamma_{1}\left(\int_{0}^{1-\tau} \varphi_{1}(t+\tau) d t+\int_{\theta}^{1} \varphi_{1}(t-\theta) d t+\tau+\theta\right)} \\
& =\bar{R} .
\end{aligned}
$$

Let $R_{1}=1+\max \left\{R^{\prime}, \bar{R}\right\}$. Then, we obtain $A(s, x) \neq x$ for $x \in \partial K_{r}$ and $s \in[0,1]$. In view of (143), (144), and homotopy invariance of the fixed point index, we obtain

$$
\begin{aligned}
i\left(T, K_{R_{1}}, K\right) & =i\left(A(1, \cdot), K_{R_{1}}, K\right)=i\left(A(0, \cdot), K_{R_{1}}, K\right) \\
& =i\left(T_{1}, K_{R_{1}}, K\right)=1 .
\end{aligned}
$$

By using additivity, we get that

$$
i\left(T, K_{R_{1}} \backslash \bar{K}_{h_{2}}, K\right)=1, \quad i\left(T, K_{h_{2}} \backslash \bar{K}_{h_{1}}, K\right)=-1 .
$$

Thus $T$ has fixed points $x_{1}$ and $x_{2}$ in $K_{R_{1}} \backslash \bar{K}_{h_{2}}$ and $K_{h_{2}} \backslash \bar{K}_{h_{1}}$, which means that $u_{1}(t)=x_{1}(t)+x_{0}(t)$ and $u_{2}(t)=x_{2}(t)+$ $x_{0}(t)$ are positive solutions of boundary value problem (5) and

$$
h_{1}<\left\|u_{1}\right\|_{[0,1]}=\left\|x_{1}\right\|<h_{2}<\left\|x_{2}\right\|=\left\|u_{2}\right\|_{[0,1]} .
$$

The proof is completed.

Corollary 22. Let $\left(H_{4}\right),\left(H_{7}\right)$, and $\left(H_{8}\right)$ hold. Assume that $0<$ $h_{1}<h_{2}$. Then, boundary value problem (5) has at least two positive solutions $u_{1}$ and $u_{2}$ such that

$$
0<\left\|u_{1}\right\|_{[0,1]}<h_{1}<\left\|u_{2}\right\|_{[0,1]} .
$$

Corollary 23. Let $\left(H_{5}\right),\left(H_{7}\right)$ and $\left(H_{8}\right)$ hold. Assume $0<h_{2}<$ $h_{1}$. Then boundary value problem (5) has at least two positive solutions $u_{1}$ and $u_{2}$ such that

$$
h_{2}<\left\|u_{1}\right\|_{[0,1]}<h_{1}<\left\|u_{2}\right\|_{[0,1]} .
$$

\section{Example}

In this section, we will present an example to illustrate our main results.
Consider the following fractional functional differential equations with delay:

$$
\begin{aligned}
& { }^{C} D^{5 / 2}\left(\left(t-\frac{1}{2}\right)^{2 C} D^{3 / 2} u(t)\right)+(t+1)^{2} \\
& +\left\{u\left(t-\frac{1}{5}\right)+u\left(t+\frac{1}{5}\right)\right\}^{1 / 3}=0, \quad t \in[0,1], \\
& { }^{C} D^{3 / 2} u(0)={ }^{C} D^{3 / 2} u(1)=\left({ }^{C} D^{3 / 2} u(0)\right)^{\prime \prime}=0,
\end{aligned}
$$

$$
\begin{aligned}
& u(t)-2 u^{\prime}(t)=\sin t, \quad t \in\left[-\frac{1}{3}, 0\right] \\
& 3 u(t)+5 u^{\prime}(t)=\cos t, \quad t \in\left[1, \frac{4}{3}\right] .
\end{aligned}
$$

By a simple computation, we can get that $b>((2-\alpha) /(\alpha-$ 1)) $a$ and

$$
\begin{aligned}
& \min _{t \in[0,1]} \frac{f(t, \mu, v)}{\mu+v}=\min _{t \in[0,1]} \frac{(t+1)^{2}+(u+v)^{1 / 3}}{u+v} \\
& =\frac{1}{u+v}+\frac{1}{(u+v)^{2 / 3}} \longrightarrow \infty
\end{aligned}
$$

as $u+v \rightarrow 0^{+}$.

Similarly, we can obtain $\min _{t \in[0,1]} f(t, \mu, \nu) /(\mu+\nu) \rightarrow 0$ as $u+v \rightarrow+\infty$.

Then, conditions $\left(H_{1}\right)$ and $\left(H_{3}\right)$ are satisfied. Then, by Theorem 15, boundary value problem (161) has at least a positive solution.

\section{Acknowledgments}

The authors sincerely thank the reviewers for their valuable suggestions and useful comments that have led to the present improved version of the original paper. This research is supported by the Natural Science Foundation of China (11071143), Natural Science Outstanding Youth Foundation of Shandong Province (JQ201119), Shandong Provincial Natural Science Foundation (ZR2012AM009, ZR2011AL007), Natural Science Foundation of Educational Department of Shandong Province (J11LA01).

\section{References}

[1] K. B. Oldham and J. Spanier, The Fractional Calculus, Academic Press, New York, NY, USA, 1974.

[2] K. S. Miller and B. Ross, An Introduction to the Fractional Calculus and Fractional Differential Equation, John Wiley \& Sons, New York, NY, USA, 1993.

[3] S. G. Samko, A. A. Kilbas, and O. I. Marichev, Fractional Integral and Derivative, Theory and Applications, Gordon and Breach, Yverdon, Switzerland, 1993.

[4] I. Podlubny, Fractional Differential Equations, Academic Press, San Diego, Calif, USA, 1999.

[5] A. A. Kilbas, H. M. Srivastava, and J. J. Trujillo, Theory and Applications of Fractional Differential Equations, Elsevier Science, Amsterdam, The Netherlands, 2006. 
[6] K. Diethelm, The Analysis of Fractional Differential Equations, Springer, Berlin, Germany, 2010.

[7] R. P. Agarwal, Y. Zhou, J. Wang, and X. Luo, "Fractional functional differential equations with causal operators in Banach spaces," Mathematical and Computer Modelling, vol. 54, no. 56, pp. 1440-1452, 2011.

[8] R. P. Agarwal, M. Benchohra, and S. Hamani, "Boundary value problems for fractional differential equations," Georgian Mathematical Journal, vol. 16, no. 3, pp. 401-411, 2009.

[9] R. P. Agarwal, Y. Zhou, and Y. He, "Existence of fractional neutral functional differential equations," Computers \& Mathematics with Applications, vol. 59, no. 3, pp. 1095-1100, 2010.

[10] V. Lakshmikantham, S. Leela, and J. V. Devi, Theory of Fractional Dynamic Systems, Cambridge Academic, Cambridge, UK, 2009.

[11] Y. Zhao, S. Sun, Z. Han, and Q. Li, “The existence of multiple positive solutions for boundary value problems of nonlinear fractional differential equations," Communications in Nonlinear Science and Numerical Simulation, vol. 16, no. 4, pp. 2086-2097, 2011.

[12] Y. Zhao, S. Sun, Z. Han, and Q. Li, "Positive solutions to boundary value problems of nonlinear fractional differential equations," Abstract and Applied Analysis, vol. 2011, Article ID 390543, 16 pages, 2011.

[13] Y. Zhao, S. Sun, Z. Han, and M. Zhang, "Positive solutions for boundary value problems of nonlinear fractional differential equations," Applied Mathematics and Computation, vol. 217, no. 16, pp. 6950-6958, 2011.

[14] W. Feng, S. Sun, Z. Han, and Y. Zhao, "Existence of solutions for a singular system of nonlinear fractional differential equations," Computers \& Mathematics with Applications, vol. 62, no. 3, pp. 1370-1378, 2011.

[15] Y. Pan, Z. Han, S. Sun, and Y. Zhao, "The existence of solutions to a system of discrete fractional boundary value problems," Abstract and Applied Analysis, vol. 2012, Article ID 707631, 15 pages, 2012.

[16] Y. Pan, Z. Han, S. Sun, and Z. Huang, "The existence and uniqueness of solutions to boundary value problems of fractional difference equations," Mathematical Sciences, vol. 6, no. 7, pp. 1-10, 2012.

[17] B. Ahmad and J. J. Nieto, "Riemann-Liouville fractional differential equations with fractional boundary conditions," Fixed Point Theory on Fixed Point Theory, Computation and Applications, vol. 13, no. 2, pp. 329-336, 2012.

[18] X. Ding, Y. Feng, and R. Bu, "Existence, nonexistence and multiplicity of positive solutions for nonlinear fractional differential equations," Journal of Applied Mathematics and Computing, vol. 40, no. 1-2, pp. 371-381, 2012.

[19] D. O’Regan and S. Staněk, "Fractional boundary value problems with singularities in space variables," Nonlinear Dynamics of Nonlinear Dynamics and Chaos in Engineering Systems, vol. 71, no. 4, pp. 641-652, 2013.

[20] T. Maraaba, D. Baleanu, and F. Jarad, "Existence and uniqueness theorem for a class of delay differential equations with left and right Caputo fractional derivatives," Journal of Mathematical Physics, vol. 49, no. 8, Article ID 083507, 11 pages, 2008.

[21] X. Li, L. Song, and J. Wei, "Positive solutions for boundary value problem of nonlinear fractional functional differential equations," Applied Mathematics and Computation, vol. 217, no. 22, pp. 9278-9285, 2011.

[22] Y. Zhao, H. Chen, and L. Huang, "Existence of positive solutions for nonlinear fractional functional differential equation,"
Computers \& Mathematics with Applications, vol. 64, no. 10, pp. 3456-3467, 2012.

[23] M. Benchohra, J. Henderson, S. K. Ntouyas, and A. Ouahab, "Existence results for fractional order functional differential equations with infinite delay," Journal of Mathematical Analysis and Applications, vol. 338, no. 2, pp. 1340-1350, 2008.

[24] Y. Zhou, F. Jiao, and J. Li, "Existence and uniqueness for $p$-type fractional neutral differential equations," Nonlinear Analysis: Theory, Methods and Applications A, vol. 71, no. 7-8, pp. 27242733, 2009.

[25] Y. Zhou, F. Jiao, and J. Li, "Existence and uniqueness for fractional neutral differential equations with infinite delay," Nonlinear Analysis: Theory, Methods and Applications A, vol. 71, no. 7-8, pp. 3249-3256, 2009.

[26] J. Wang, Y. Zhou, and W. Wei, "A class of fractional delay nonlinear integrodifferential controlled systems in Banach spaces," Communications in Nonlinear Science and Numerical Simulation, vol. 16, no. 10, pp. 4049-4059, 2011.

[27] A. Babakhani, D. Baleanu, and R. P. Agarwal, "The existence and uniqueness of solutions for a class of nonlinear fractional differential equations with infinite delay," Abstract and Applied Analysis, vol. 2013, Article ID 592964, 8 pages, 2013.

[28] Y. Zhou, Y. Tian, and Y.-Y. He, "Floquet boundary value problem of fractional functional differential equations," Electronic Journal of Qualitative Theory of Differential Equations, vol. 50, pp. 1-13, 2010.

[29] L. Zhang, B. Ahmad, G. Wang, and R. P. Agarwal, "Nonlinear fractional integro-differential equations on unbounded domains in a Banach space," Journal of Computational and Applied Mathematics, vol. 249, pp. 51-56, 2013.

[30] Y. Li, S. Sun, D. Yang, and Z. Han, "Three-point boundary value problems of fractional functional differential equations with delay," Boundary Value Problems, vol. 2013, article 38, 15 pages, 2013.

[31] C. Bai, "Existence of positive solutions for a functional fractional boundary value problem," Abstract and Applied Analysis, vol. 2010, Article ID 127363, 13 pages, 2010.

[32] Z. Ouyang, Y. Chen, and S. Zou, "Existence of positive solutions to a boundary value problem for a delayed nonlinear fractional differential system," Boundary Value Problems, vol. 2011, Article ID 475126, 17 pages, 2011.

[33] S. B. Norkin, Differential Equations of the Second Order with Retarded Argument, vol. 31 of Translation of Mathematical Monographs, American Mathematical Society, Providence, RI, USA, 1972.

[34] C. Bai and J. Ma, "Eigenvalue criteria for existence of multiple positive solutions to boundary value problems of second-order delay differential equations," Journal of Mathematical Analysis and Applications, vol. 301, no. 2, pp. 457-476, 2005.

[35] D. J. Guo and V. Lakshmikantham, Nonlinear Problems in Abstract Cone, Academic Press, Orlando, Fla, USA, 1988.

[36] K. Deimling, Nonlinear Functional Analysis, Springer, Berlin, Germany, 1985. 


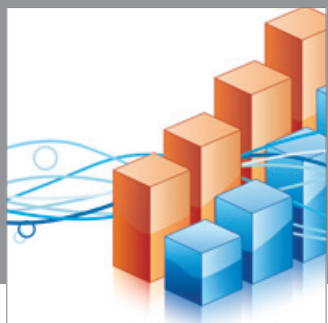

Advances in

Operations Research

mansans

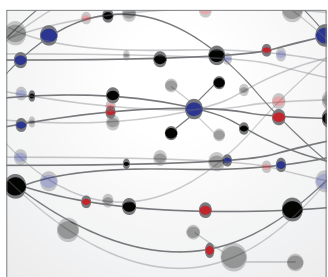

The Scientific World Journal
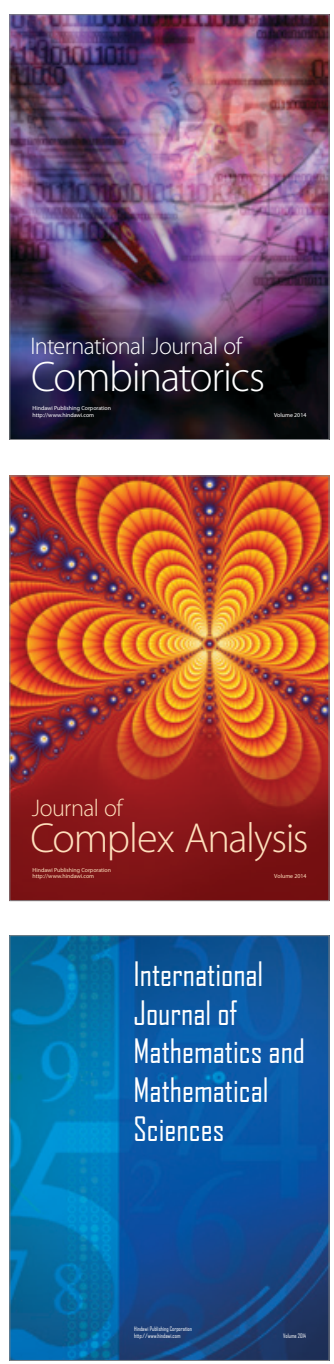
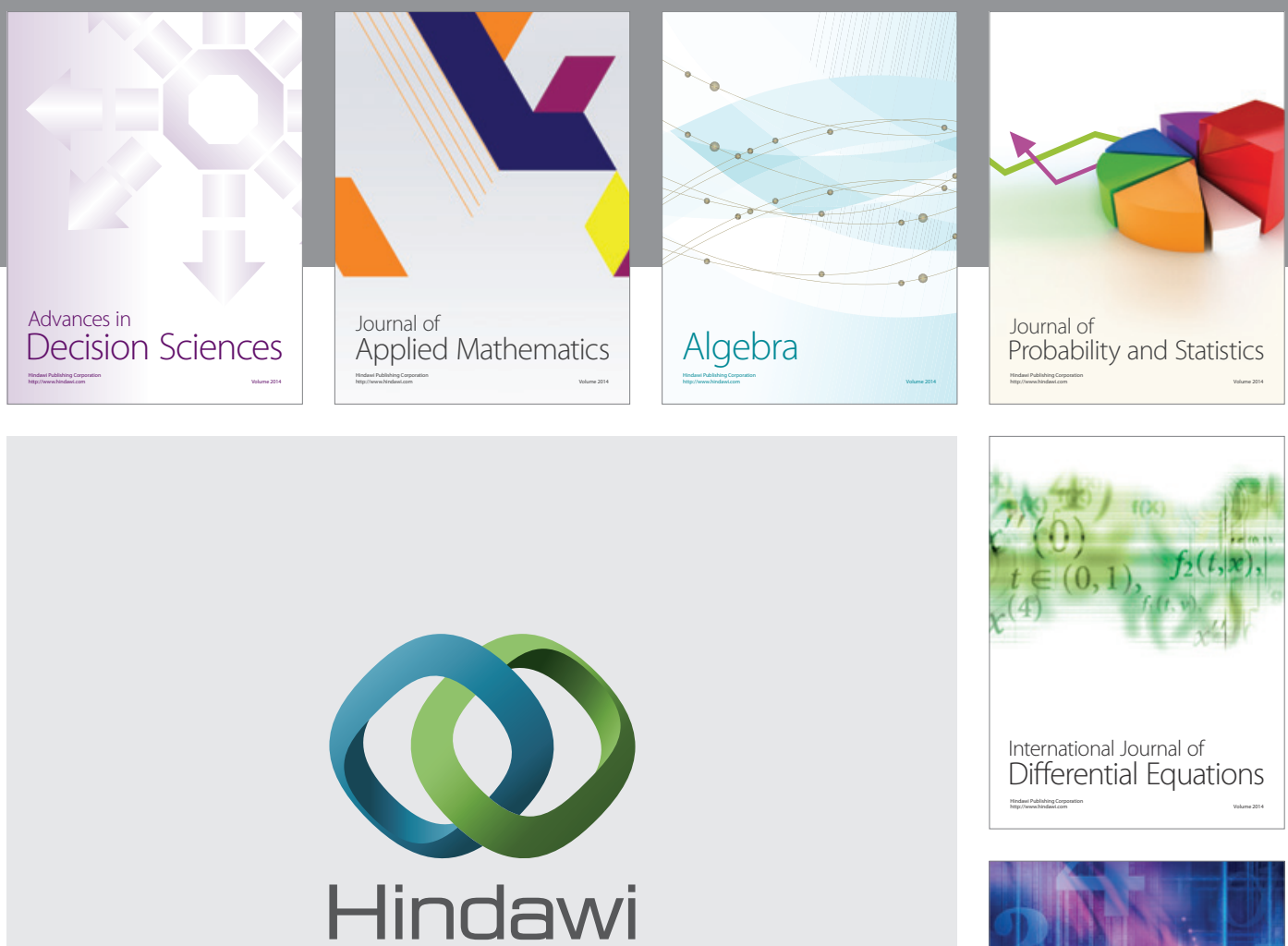

Submit your manuscripts at http://www.hindawi.com
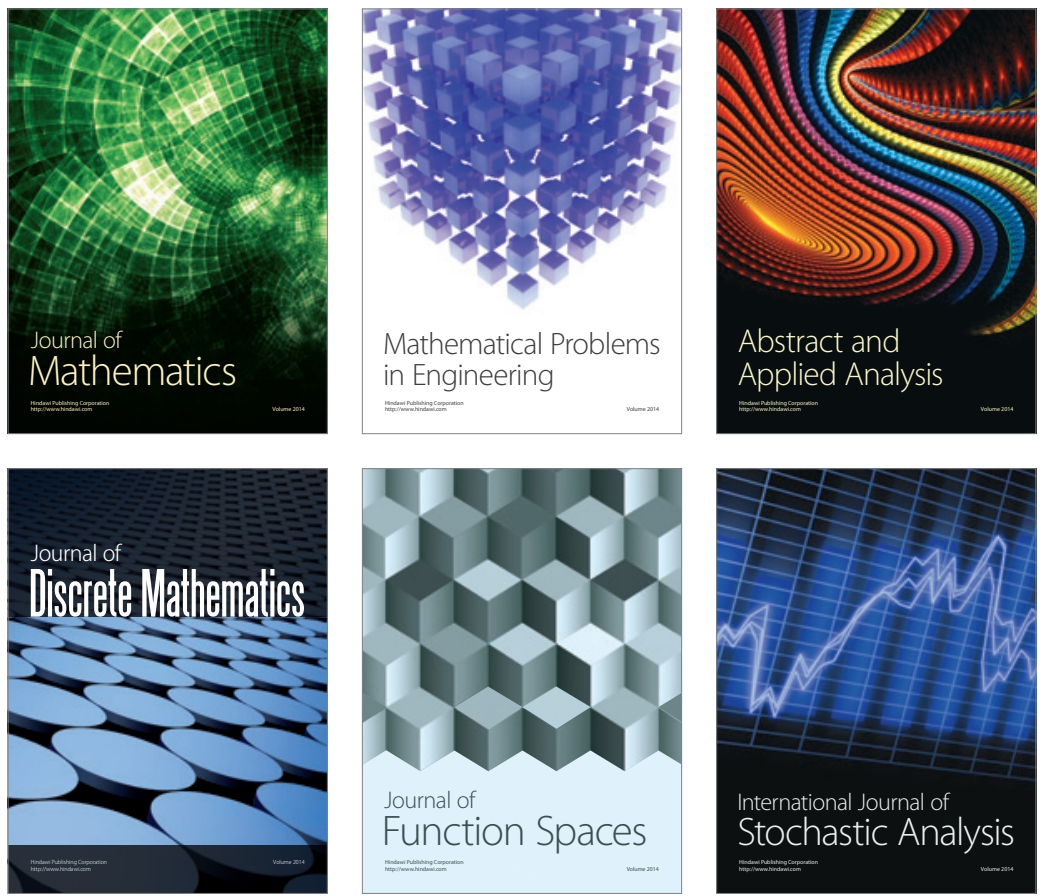

Journal of

Function Spaces

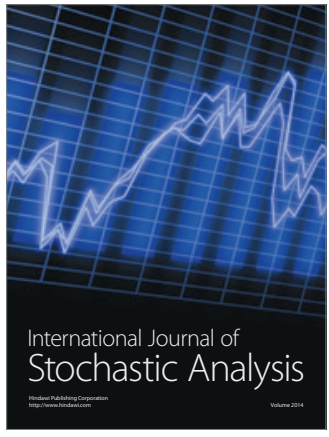

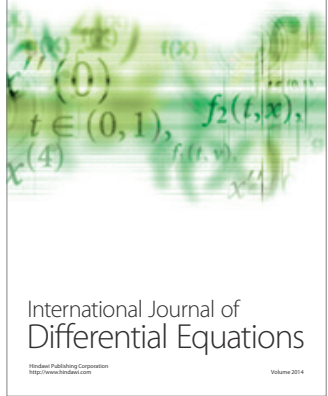
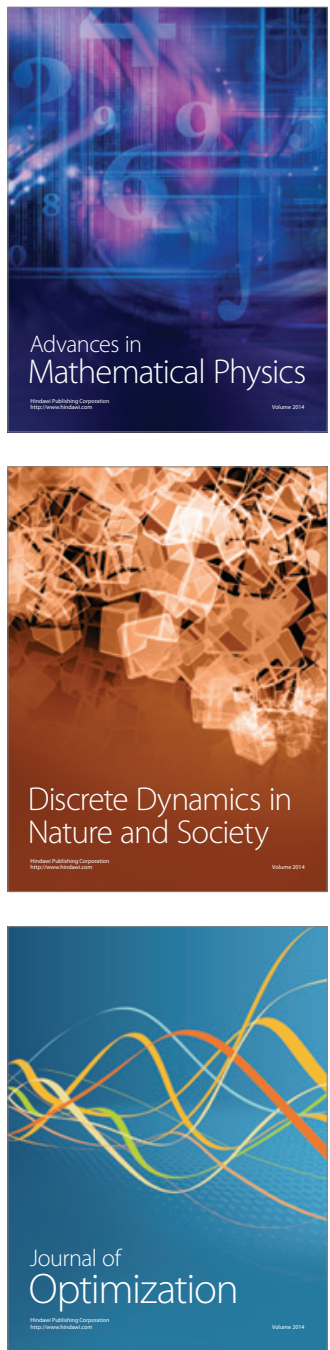